\title{
Effective theory of flavor for Minimal Mirror Twin Higgs
}

\author{
Riccardo Barbieri, ${ }^{a}$ Lawrence J. Hall ${ }^{b, c}$ and Keisuke Harigaya ${ }^{b, c}$ \\ ${ }^{a}$ Scuola Normale Superiore and INFN, \\ Pisa, Italy \\ ${ }^{b}$ Department of Physics, University of California, \\ Berkeley, California 94720, U.S.A. \\ ${ }^{c}$ Theoretical Physics Group, Lawrence Berkeley National Laboratory, \\ Berkeley, California 94720, U.S.A. \\ E-mail: riccardo.barbieri@sns.it, ljhall@lbl.gov, \\ keisukeharigaya@berkeley.edu
}

Abstract: We consider two copies of the Standard Model, interchanged by an exact parity symmetry, $P$. The observed fermion mass hierarchy is described by suppression factors $\epsilon^{n_{i}}$ for charged fermion $i$, as can arise in Froggatt-Nielsen and extra-dimensional theories of flavor. The corresponding flavor factors in the mirror sector are $\epsilon^{\prime n_{i}}$, so that spontaneous breaking of the parity $P$ arises from a single parameter $\epsilon^{\prime} / \epsilon$, yielding a tightly constrained version of Minimal Mirror Twin Higgs, introduced in our previous paper. Models are studied for simple values of $n_{i}$, including in particular one with $\mathrm{SU}(5)$-compatibility, that describe the observed fermion mass hierarchy. The entire mirror quark and charged lepton spectrum is broadly predicted in terms of $\epsilon^{\prime} / \epsilon$, as are the mirror QCD scale and the decoupling temperature between the two sectors. Helium-, hydrogen- and neutron-like mirror dark matter candidates are constrained by self-scattering and relic ionization. In each case, the allowed parameter space can be fully probed by proposed direct detection experiments. Correlated predictions are made as well for the Higgs signal strength and the amount of dark radiation.

Keywords: Beyond Standard Model, Cosmology of Theories beyond the SM

ARXIV EPRINT: 1706.05548 


\section{Contents}

1 Introduction 1

2 Minimal flavor hierarchy 2

3 SU(5)-compatible model 4

3.1 Mass spectrum of mirror fermions 4

3.2 Higgs signal 6

3.3 Mirror dark matter 7

3.3.1 Dark matter candidates 8

$\begin{array}{lll}\text { 3.3.2 Direct detection via Higgs exchange } & 10\end{array}$

3.3.3 Constraint on $(\mathrm{He})_{*}^{\prime}$ dark matter: region (a) of figure $4 \quad 11$

3.3.4 Constraints on $H^{\prime} / H_{*}^{\prime}$ dark matter: regions (b) and (f) of figure $4 \quad 13$

3.3.5 Constraints on $n^{\prime}$ dark matter: regions (c), (d) and (e) of figure $4 \quad 15$

$\begin{array}{lll}3.3 .6 & \text { Mirror and SM matter asymmetries } & 15\end{array}$

3.3.7 Possibility of mirror nucleosynthesis 16

3.4 Dark radiation 16

$\begin{array}{lll}\text { 3.4.1 Generic decoupling temperature } & 17\end{array}$

3.4.2 Decoupling temperature from Higgs exchange 19

3.4.3 Decoupling temperature from kinetic mixing 22

4 Variant models $\quad 22$

5 Conclusions 23

A Minimal flavor hierarchy from extra dimensions $\quad 25$

$\begin{array}{ll}\text { B Scaling law } & 27\end{array}$

C Evidence for the minimal flavor hierarchy 28

D Mirror matter asymmetry for $m_{u^{\prime}} \sim m_{d^{\prime}} \quad 28$

E Mirror recombination with electron capture 30

\section{Introduction}

A Mirror Sector, an identical copy of the Standard Model (SM) [1, 2], is currently of considerable interest. Two key results follow from introducing an approximate spacetime parity symmetry, $P$, that exchanges the two sectors. First, dark matter may be mirror baryons [3] with a density expected to be the same order as the baryon density. Second, the SM Higgs boson can be understood as a pseudo-Goldstone boson via the Twin Higgs mechanism [4], even though it has order unity couplings, with a modest amount of fine-tuning. 
A key question is how $P$ is broken. Simple schemes that have $P$ broken only via a Higgs mass term suffer from two key problems. First, the theory is excluded from excessive dark radiation from the mirror sector. Second, in such schemes mirror dark matter is in part hydrogen-like, with parameters that are excluded by self scattering. Further there is the question of the origin of this $P$-breaking Higgs mass.

Recently we introduced Minimal Mirror Twin Higgs (MMTH) [5], where $P$ is broken only in the Yukawa couplings. In the absence of an exotic cosmological history after the two sectors decouple (see $[6,7]$ for examples of such history), we showed that $P$-breaking in the Yukawa couplings is a necessity to solve the dark radiation problem, even if additional interactions allow the decoupling temperature to be arbitrary. Also, a variety of candidates for mirror dark matter are possible that are not excluded and predict rich phenomenology. ${ }^{1}$ Furthermore, in MMTH a $P$-breaking Higgs mass term, necessary for the Twin Higgs mechanism, is generated by 1-loop radiative corrections. We showed that MMTH has correlated signals in Higgs decays, direct detection of dark matter and dark radiation, over a region of parameter space where the fine-tuning for the electroweak scale is $10-50 \%$.

Nevertheless, MMTH itself leads to two questions: what is the origin of $P$ breaking in the Yukawa sector? Given the large number of parameters in the Yukawa sector, how predictive can the theory be? In section 2 we introduce a minimal flavor hierarchy for MMTH, defined in eq. (2.2), where the mirror fermion spectrum is predicted to leading order in terms of a single parameter $\epsilon^{\prime} / \epsilon$. Such hierarchies arise in Froggatt-Nielsen theories [9] with an Abelian flavor symmetry spontaneously broken by a small parameter $\epsilon$, as shown in eq. (2.3), and they can also arise in extra-dimensional theories of flavor [10].

In section 3 we study in detail the resulting Higgs, dark radiation and dark matter signals in a particular model where the powers of $\epsilon$, the Froggatt-Nielsen charges, are compatible with SU(5) unification. We give predictions for the Higgs signal strength and the amount of dark radiation, and focus on the nature and signals of mirror dark matter. We show regions for hydrogen- and helium-like dark matter that are currently allowed by direct detection, self-scattering and relic ionization limits, and discover that there is a significantly larger parameter region for mirror neutron dark matter that is currently much less constrained. We find that almost all regions for these dark matter candidates that are presently allowed can be probed by direct detection in experiments under way.

Variant models are briefly discussed in section 4. Although the predictions differ in detail, the broad picture is the same: all models with a single parameter describing charged fermion mass hierarchies are highly constrained by data. Conclusions are drawn in section 5 and several calculations and details are presented in appendices A to E.

\section{Minimal flavor hierarchy}

A key feature of the quark and charged lepton masses is their large hierarchies. Any theory of flavor should incorporate a set of parameters $\epsilon_{a} \ll 1$ to describe these hierarchies. Within the context of MMTH it is interesting to explore the possibility that the only breaking of

\footnotetext{
${ }^{1}$ The possibility to address both the dark matter and the dark radiation problems by Yukawa couplings of the light mirror fermions larger than the SM ones is proposed in [8].
} 
$P$ arises spontaneously from a difference between these hierarchy parameters in the two sectors, $\epsilon_{a}^{\prime} \neq \epsilon_{a}$. A general form for the $3 \times 3$ up, down and charged lepton Yukawa matrices in the two sectors in the effective theory below $\Lambda$ is

$$
y_{i j}\left(\epsilon_{a}\right)=\sum \lambda_{i j}^{a} \epsilon_{a}^{n_{i j}} \quad y_{i j}^{\prime}\left(\epsilon_{a}\right)=\sum \lambda_{i j}^{a} \epsilon_{a}^{\prime n_{i j}}
$$

where $\lambda_{i j}^{a}$ are order unity and the same in each sector. The powers $n_{i j}^{a}$ vary between theories, and the summation indicates that several such terms may be relevant for any $i j$.

In this paper we provide sharp predictions for MMTH by focussing on a simple scheme for flavor symmetry breaking in the effective theory below $\Lambda$, with a single hierarchy parameter in each sector so that the label $a$ may be dropped. In this "Minimal Flavor Hierarchy" each Yukawa matrix element is dominated by a single term of the form

$$
y_{i j}=\epsilon^{n_{i}} \lambda_{i j} \epsilon^{\bar{n}_{j}} \quad y_{i j}^{\prime}=\epsilon^{\prime n_{i}} \lambda_{i j} \epsilon^{\prime \bar{n}_{j}} .
$$

With this structure, the coupling to the $i(j)$ fermions on the left (right) receives a suppression of the hierarchy parameter to the $n_{i}\left(\bar{n}_{j}\right)$ power. We stress that $P$ forces $n_{i}, \bar{n}_{j}$ and $\lambda_{i j}$ to be the same in the two sectors, while the spontaneous breaking of $P$ arises only via the single parameter $\epsilon^{\prime} / \epsilon \neq 1$, which is constrained by data to typically be in the range of $2-3$.

What is the UV completion of the theory that leads to the structure of (2.2) in the effective theory at the TeV scale? Above $\Lambda$ the twin Higgs sector must be UV completed, for example in a composite Higgs [11-18] or supersymmetric theory [19-23]. Without addressing this completion, we can still discuss how the flavor breaking spurions $\epsilon^{n_{i}}, \epsilon^{\bar{n}_{j}}$ arise at high energies. Possibilities include Frogatt-Nielsen (FN) [9] and extra-dimensional theories [10, 24].

We consider a FN theory with a U(1) flavor symmetry in each sector spontaneously broken by $\left\langle\phi^{\prime}\right\rangle \neq\langle\phi\rangle$, which is the only breaking of $P$ in the theory. The flavor structure of $(2.2)$ results when the fermion charges $\left(Q_{i}, \bar{Q}_{j}\right)$ are chosen to be $\left(n_{i}, \bar{n}_{j}\right)$ and, for example, $\epsilon=\langle\phi\rangle / M$ and $\epsilon^{\prime}=\left\langle\phi^{\prime}\right\rangle / M$, where $M$ is the mass scale suppressing higher-dimensional operators which have order unity couplings $\lambda_{i j}$. In summary

$$
\epsilon=\frac{\langle\phi\rangle}{M}, \quad \epsilon^{\prime}=\frac{\left\langle\phi^{\prime}\right\rangle}{M}, \quad\left(n_{i}, \bar{n}_{j}\right)=\left(Q_{i}, \bar{Q}_{j}\right) .
$$

The non-degeneracies between heavy FN fermions of the two sectors must not be so large that the Twin Higgs mechanism is upset. While there are many such models, they are greatly restricted since they must reproduce the known charged fermion masses. We find it convenient to take the charges to be integral and $\epsilon$ close to the Cabibbo angle, and study the predictions of three such models in detail.

Small flavor parameters can arise from wavefunctions of zero-modes in extra dimensions [24]. The analysis of this paper is based entirely on the Yukawa structure of (2.2) can it apply to extra-dimensional theories as well as 4D FN theories? If the Higgs field is spread out in the bulk and fermion wavefunctions are Gaussian, as in [24], then the Yukawa matrix elements do not have the form of (2.2) as the overlap integral of the two fermion 
wavefunctions does not factor into a suppression factor for each fermion. However, if the Higgs is localized in the bulk at $y_{H}$, the structure of $y_{i j}$ in (2.2) arises for any form of the wavefunctions of the fermions in the bulk, with $\epsilon^{n_{i}}=\psi_{i}\left(y_{H}\right)$ and $\epsilon^{\bar{n}_{j}}=\psi_{j}\left(y_{H}\right)$, and $\lambda_{i j}$ is the brane-localized coupling at $y_{H}[10]$. However, it is not clear what spontaneous breaking in the higher-dimensional set up would lead to $\epsilon^{\prime} \neq \epsilon$ while leaving the powers $n_{i}, \bar{n}_{j}$ the same in both sectors.

In appendix A we give two examples of how this could happen. In one example, the fermions of the two sectors each live on orthogonal $S^{1} / Z_{2}$ spaces that intersect at the Higgs brane in a $2 \mathrm{D}$ bulk. The parity $P$ interchanges these two spaces and is spontaneous broken by compactification to give different lengths, $L^{\prime} \neq L$. We find the flavor structure of $(2.2)$ is reproduced with

$$
\epsilon=e^{-\mu L}, \quad \quad \epsilon^{\prime}=e^{-\mu L^{\prime}}, \quad\left(n_{i}, \bar{n}_{j}\right)=\left(\frac{M_{i}}{\mu}, \frac{\bar{M}_{j}}{\mu}\right),
$$

where $M_{i}$ and $\bar{M}_{j}$ are bulk masses of the fermions and $\mu$ is an arbitrary scale which we choose to give $\epsilon$ close to the Cabibbo angle.

\section{$3 \mathrm{SU}(5)$-compatible model}

In this section we investigate the prediction of a model with a $\mathrm{U}(1)$ flavor symmetry. We consider a model consistent with the embedding of quarks and leptons into SU(5) multiplets. We discuss the mass spectrum of mirror fermions, its effect on the Higgs signal, dark matter phenomenology, and the amount of the dark radiation. We expect the main features of the results to be similar for other U(1) charge assignment as long as the observed fermions mass hierarchy is well reproduced, as in the two other models briefly discussed in section 4 .

\subsection{Mass spectrum of mirror fermions}

In this section we study U(1) flavor charges of fermions consistent with SU(5) [25-27]:

$$
Q, \bar{u}, \bar{e}:(4,2,0), \quad \bar{d}, L:(4,3,3) .
$$

The three numbers in each parenthesis denote charges of the first, second and third generation fermions, respectively. Using this structure in eqs. (2.2) and (2.3), the Yukawa couplings of the Standard Model (SM) fermions are given by

$$
\begin{aligned}
y_{t} & \sim 1+O\left(\epsilon^{4}\right), & y_{c} & \sim \epsilon^{4}\left(1+O\left(\epsilon^{4}\right)\right), & & y_{u} \sim \epsilon^{8}\left(1+O\left(\epsilon^{4}\right)\right) \\
y_{b} & \sim \epsilon^{3}\left(1+O\left(\epsilon^{2}\right)\right), & & y_{s} \sim \epsilon^{5}\left(1+O\left(\epsilon^{2}\right)\right), & & y_{d} \sim \epsilon^{8}\left(1+O\left(\epsilon^{2}\right)\right) \\
y_{\tau} & \sim \epsilon^{3}\left(1+O\left(\epsilon^{2}\right)\right), & & y_{\mu} \sim \epsilon^{5}\left(1+O\left(\epsilon^{2}\right)\right), & & y_{e} \sim \epsilon^{8}\left(1+O\left(\epsilon^{2}\right)\right),
\end{aligned}
$$

where $\epsilon=\langle\phi\rangle / M$ and order unity coefficients from the $\lambda_{i j}$ are omitted. Note that there is a correction of $O\left(\epsilon^{2}\right)$ or $O\left(\epsilon^{4}\right)$ to the leading order $\epsilon^{n}$ terms. The derivation of the leading and correction terms are given in appendix B for down-type quarks. The quality of the $\mathrm{SU}(5)$ model as an explanation of the flavour hierarchy is exhibited in appendix C. 


\begin{tabular}{|c|c|c|c|c|c|c|c|c|}
\hline$y_{e}$ & $y_{\mu}$ & $y_{\tau}$ & $y_{d}$ & $y_{u}$ & $y_{s}$ & $y_{c}$ & $y_{b}$ & $y_{t}$ \\
\hline $2.8 \times 10^{-6}$ & $5.9 \times 10^{-4}$ & $1.0 \times 10^{-2}$ & $1.6 \times 10^{-5}$ & $7.4 \times 10^{-6}$ & $3.1 \times 10^{-4}$ & $3.6 \times 10^{-3}$ & $1.6 \times 10^{-2}$ & 0.99 \\
\hline
\end{tabular}

Table 1. Yukawa couplings of the SM fermions at the renormalization scale $\mu=m_{Z}$.

For a fermion $f$ with a dependence $y_{f} \sim \epsilon^{n}\left(1+O\left(\epsilon^{m}\right)\right)$, the ratio of the Yukawa couplings of the corresponding mirror fermion to that of the SM fermion, at the same scale above both masses, is given by

$$
\frac{y_{f^{\prime}}}{y_{f}}=\left(\frac{\epsilon^{\prime}}{\epsilon}\right)^{n}\left(1+\delta_{f} \epsilon^{\prime m}-\delta_{f} \epsilon^{m}\right)
$$

where $\delta_{f}$ depend on the $\lambda_{i j}$ and hence are unknown $O(1)$ constants. It should be noted that the top quark has $n=0$, and hence the SM and the mirror top yukawa couplings are the same (up to small corrections of relative order $\epsilon^{\prime 4}, \epsilon^{4}$ ) which is required to suppress a too large correction to the Higgs mass term [28]. We use values of the SM Yukawa couplings shown in table 1 at the renormalization scale $\mu=m_{Z}$ [29]. In figure 1 , we show the masses of mirror fermions, including renormalization by the strong coupling. The bands show the uncertainty due to the unknown constants $\delta_{f}$, and correspond to $\left|\delta_{f}\right|<1$. The SM Yukawa couplings $y_{u}$ and $y_{d}$ suffer uncertainties of $30 \%$ and $10 \%$, but we assume central values in figure 1.

The mass spectrum of the mirror particles also depends on the dynamical scale of mirror QCD, $\Lambda_{Q C D}^{\prime}$. To estimate $\Lambda_{Q C D}^{\prime}$ and the mirror QCD phase transition temperature $T_{c}^{\prime}$, we first take the mirror top quark mass to be $4 m_{t}$, corresponding to $v^{\prime} / v=4$, and the other mirror quark masses to be $50 \mathrm{GeV}$, and solve the renormalization group running of the mirror QCD coupling constant. We then find the renormalization scale such that $6 / g_{3}^{\prime 2}=3.2$, we match the scale with the inverse of the lattice spacing and we estimate $T_{c}^{\prime}$ based on the lattice calculation in [30]. To estimate $T_{c}^{\prime}$ for generic quark masses, we then use the scaling by the one-loop renormalization group equation. The mirror QCD phase transition temperature is given by

$$
\begin{aligned}
T_{c}^{\prime} & \simeq 2.3 \mathrm{GeV}\left(\frac{m_{t^{\prime}}}{690 \mathrm{GeV}}\right)^{2 / 33} \prod_{q=d, s, b, u, c}\left(\frac{m_{q^{\prime}}}{50 \mathrm{GeV}}\right)^{2 / 33} \\
& \simeq 2.1 \mathrm{GeV}\left(\frac{v^{\prime} / v}{4}\right)^{4 / 11}\left(\frac{\epsilon^{\prime} / \epsilon}{2.5}\right)^{56 / 33} .
\end{aligned}
$$

Note that the last expression does not depend on the $\delta_{f}$ 's, as they should be cancelled with each other in the determinant of the mass matrix.

In the following sections we consider $\epsilon^{\prime} / \epsilon$ in the range of 2-3, and find that experimental constraints will further reduce the allowed range. This range gives an origin for the needed breaking of Parity in the Higgs potential via the difference $y_{f}^{\prime} \neq y_{f}$ in the Yukawa couplings of the light fermions [5] as well as the small difference between $y_{t}$ and $y_{t}^{\prime}$.

We comment on the effect of the mass splitting between the heavy FN fields, which are introduced to generate the structure in eq. (2.3). We first consider the case where none of the masses of heavy fermions vanishes for $\epsilon=0$, which we assume in this paper. Through 
the mixing between fermions, a small mass difference of $m^{\prime} / m=1+O\left(\epsilon^{\prime 2}\right)$ is expected, where $m$ and $m^{\prime}$ are the mass scale of the heavy SM FN fermions and that of the heavy mirror FN fermions, respectively, Although a difference between the gauge couplings $g_{3,2,1}$ and $g_{3,2,1}^{\prime}$ is induced due to a threshold effect, its effects on the breaking of the Parity in the Higgs potential is negligibly small. The difference between $g_{3}$ and $g_{3}^{\prime}$ does not affect the estimation of $\Lambda_{Q C D}^{\prime}$ and hence of $T_{c}^{\prime}$ at the one-loop level, as the product of the fermion masses including light fermions are not affected by the mixing, and eq. (3.4) remains intact.

It is also possible that some of the heavy fermion masses vanishes for $\epsilon=0$. In this case, $\epsilon \neq \epsilon^{\prime}$ directly affects the mass splitting of those heavy fermions, and a mass splitting of $m^{\prime} / m \sim\left(\epsilon^{\prime} / \epsilon\right)^{n}$ is expected. The Parity breaking threshold correction to the gauge coupling constant is given by

$$
\frac{\alpha_{i}^{\prime}-\alpha_{i}}{\alpha_{i}} \simeq \frac{\alpha_{i}}{2 \pi} N \ln \left(\frac{\epsilon^{\prime}}{\epsilon}\right)^{n}
$$

where $N$ is the multiplicity of the FN fermions with a large mass splitting. As long as $\alpha_{2(3)}^{\prime}-\alpha_{2(3)} / \alpha \lesssim 0.2(0.5)$, the Parity breaking effect on the Higgs potential is small [5], which requires $N n \lesssim 40$. A displacement of $T_{c}^{\prime}$ as well as the mirror electromagnetic gauge coupling is to be expected, which affect the amount of the dark radiation and the constraint on dark matter. See appendix A for analogous considerations when the Minimal Flavor Hierarchy arises from extra dimensions.

\subsection{Higgs signal}

In Twin Higgs models, the signal of the SM-like Higgs, $h$, is affected in two ways. First, $h$ is an admixture of the two original doublets $H$ and $H^{\prime}$,

$$
h=c_{\gamma} H+s_{\gamma} H^{\prime}, \quad s_{\gamma} \equiv \sin \gamma \simeq v / v^{\prime},
$$

so that the couplings between $h$ and two SM particles are reduced by a factor of $c_{\gamma}$. Second, $h$ also couples to a pair of mirror particles, so that it will decay to mirror fermions lighter than $m_{h} / 2$ via the interaction

$$
\mathcal{L} \supset-y_{f^{\prime}} H^{\prime} f_{L}^{\prime} \bar{f}_{R}^{\prime} \rightarrow-\frac{v}{\sqrt{2} v^{\prime}} y_{f^{\prime}} h f_{L}^{\prime} \bar{f}_{R}^{\prime}=-\frac{v m_{f^{\prime}}}{\sqrt{2} v^{\prime 2} \delta_{f^{\prime}, m_{h}}} h f_{L}^{\prime} \bar{f}_{R}^{\prime} .
$$

Here, $\delta_{f^{\prime}, \mu} \equiv y_{f^{\prime}}\left(m_{f^{\prime}}\right) / y_{f^{\prime}}(\mu)$ encodes the effect of renormalization between a scale $\mu$ and $m_{f^{\prime}}$. These decays lead to an invisible branching ratio for $h$

$$
\operatorname{Br}_{\mathrm{inv}}=\operatorname{Br}\left(h \rightarrow f^{\prime} \bar{f}^{\prime}\right) \simeq 0.1 \times\left(\frac{3}{v^{\prime} / v}\right)^{4} \sum_{f^{\prime}, 2 m_{f^{\prime}}<m_{h}} \frac{N_{f^{\prime}}}{3}\left(\frac{m_{f^{\prime}}}{10 \mathrm{GeV}}\right)^{2} \delta_{f^{\prime}, m_{h}}^{-2}
$$

where phase space has been neglected. The invisible branching ratio, together with the reduction of the Higgs coupling to SM particles, results in a universal deviation from unity of the Higgs signal-strengths at colliders into any SM final state,

$$
1-\mu=1-c_{\gamma}^{2}\left(1-\mathrm{Br}_{\mathrm{inv}}\right) \simeq s_{\gamma}^{2}+\mathrm{Br}_{\mathrm{inv}} .
$$



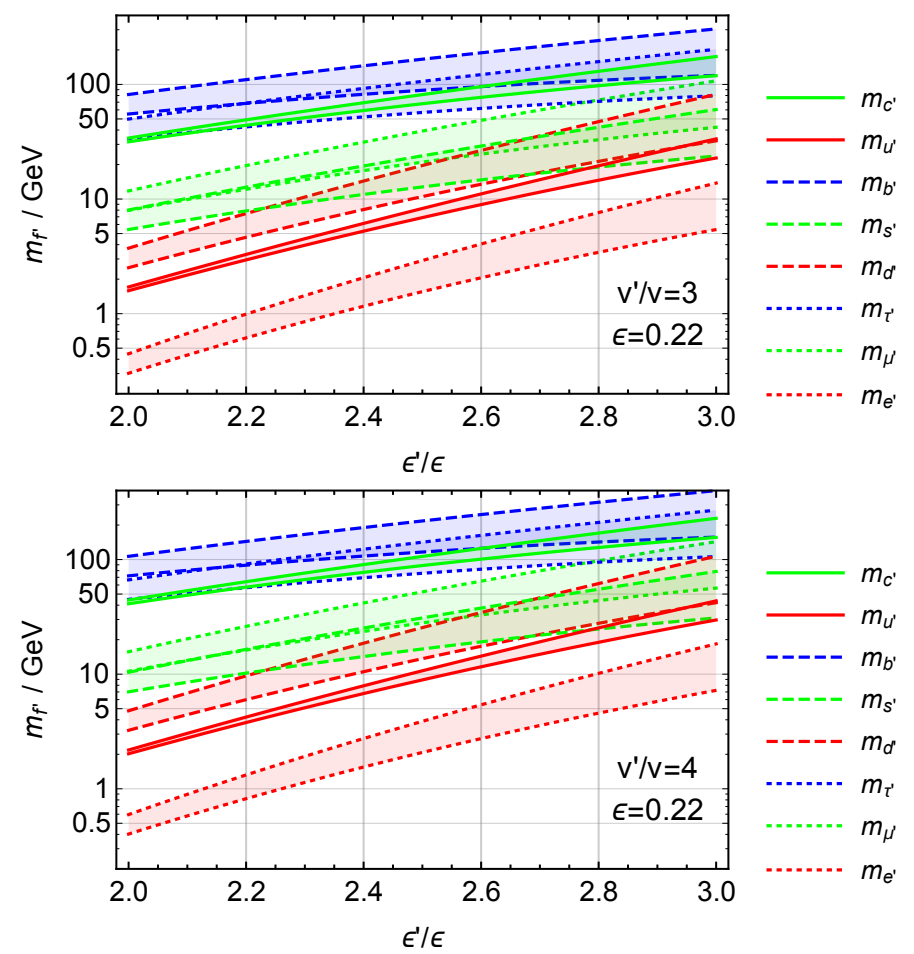

Figure 1. The mass spectrum of mirror fermions following from (3.2) and (3.3). The shaded bands, showing deviations from the simple scaling law, correspond to $\left|\delta_{f}\right|<1$. Central values are taken for SM Yukawa couplings.

In figure 2 , we show predictions on $1-\mu$ for $v^{\prime} / v=4$ and 3 . The value of $\delta$ denotes the maximum absolute value of $\delta_{f}$ we allow. We choose the sign and value of each $\delta_{f}$ so that $\mu$ becomes as large as possible. Specifically, we first try $\delta_{f}=\delta$, and see if $m_{f^{\prime}}>m_{h} / 2$. If so, we choose $\delta_{f}$ to be $\delta$. If not, we choose $\delta_{f}=-\delta$. The figure shows that $1-\mu$ can be smaller than the experimental bound, $\mu>0.75$ [31] for ranges of $\epsilon^{\prime} / \epsilon$ that depend on $v^{\prime} / v$ and $\delta$. Here we have adopted the constraint on the gluon fusion channel, as it has the smallest uncertainty. $\epsilon^{\prime} / \epsilon \lesssim 2.2$ is excluded because the mirror charm quark becomes lighter than $m_{h} / 2$.

\subsection{Mirror dark matter}

The lightest mirror baryon and the lightest mirror charged particle are stable, and may compose the dark matter of the universe. We assume that the mirror sector also has nonzero matter asymmetry and that the asymmetric component of mirror matter explains the observed dark matter density. Most of the discussion in this section is applicable to generic mirror world scenarios. Dark matter phenomenology in the mirror world scenario with $y=y^{\prime}$ is discussed in $[32,33]$ and more recently in $[34,35]$. For reviews of models producing similar/equal asymmetries in standard matter and in dark matter see [36-38] and references therein. As an example of a recent specific proposal in the context of twin Higgs models see [39]. 

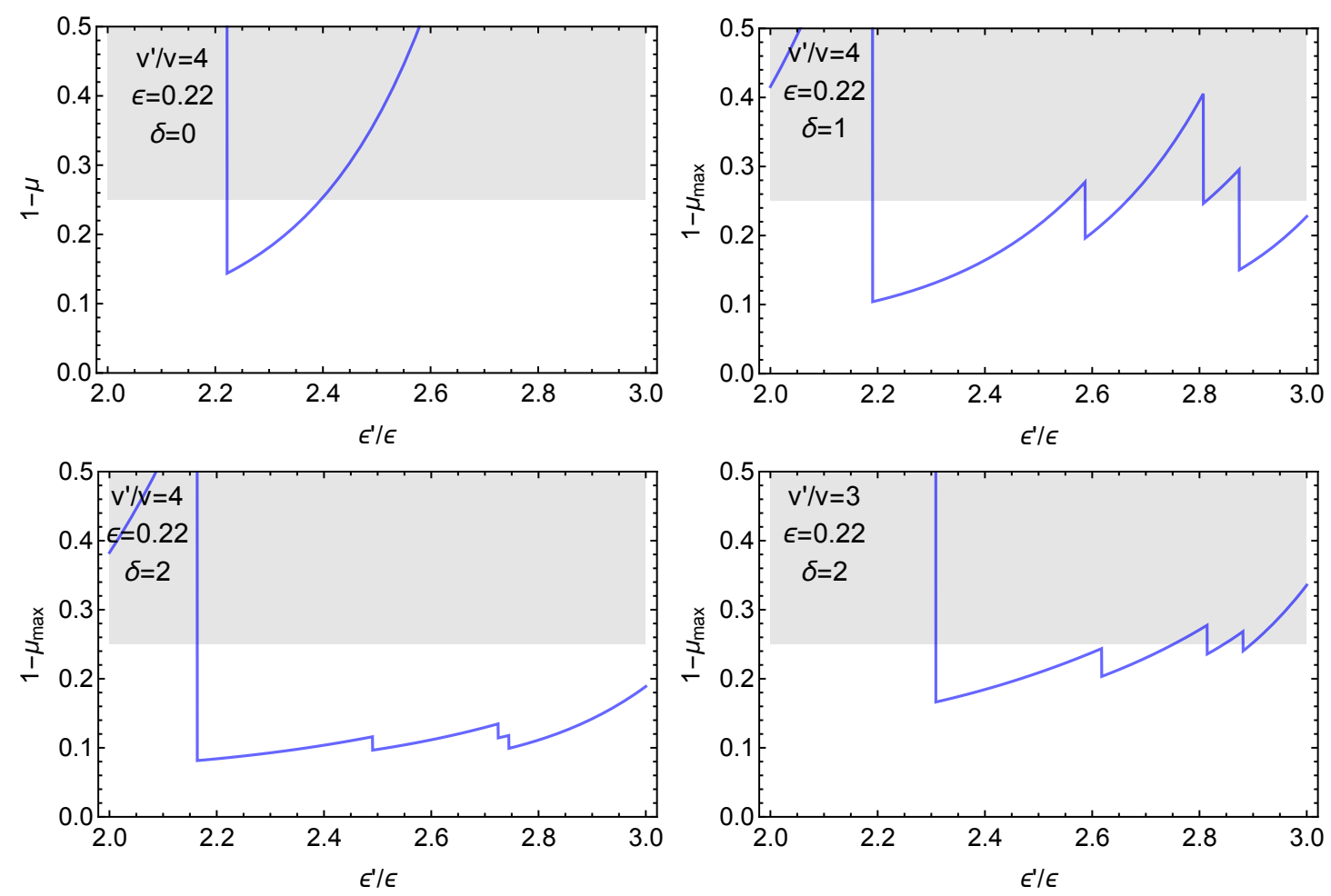

Figure 2. Prediction for the Higgs signal strength. Panels with $\delta=1,2$ have the mass spectrum of mirror fermions chosen to minimize the invisible decay of the Higgs. Decays to $c^{\prime}$ exclude $\epsilon^{\prime} / \epsilon$ less than about 2.2 .

\subsubsection{Dark matter candidates}

The second and third generation mirror fermions decay into the first generation, so only the mirror up quark, down quark or electron may be stable. The dark matter candidate depends on the mass relation between them. In the left panel of figure 3 we show the masses of $d^{\prime}, u^{\prime}$ and $e^{\prime}$ : solid, dashed and dotted lines show ranges with $\left|\delta_{f}\right| \leq 0,1$ and 2 , respectively, and uncertainties of the SM $u$ and $d$ Yukawa couplings, which we take to be $30 \%$ and $10 \%$, are included. The right panel of figure 3 shows the maximum value of $m_{d^{\prime}}$ allowed by the Higgs signal strength, for values of $\delta$ described in the caption.

In most of the parameter space $m_{e^{\prime}}<m_{u^{\prime}}+m_{d^{\prime}}$, so that the mirror electron is stable. Depending on $m_{u^{\prime}}, m_{d^{\prime}}$ there are four candidates for the lightest baryon: $B_{u u u}^{\prime}, B_{u u d}^{\prime}, B_{u d d}^{\prime}, B_{d d d}^{\prime}$. The $B_{u u u}^{\prime}, B_{d d d}^{\prime}$ states are spin $3 / 2$ and have an additional strong interaction contribution to their masses, $\Delta \sim T_{c}^{\prime}$, compared to the spin $1 / 2$ states $B_{u u d}^{\prime}, B_{u d d}^{\prime}$. From figure 3 we see that there is a large region with $m_{d^{\prime}}>m_{u^{\prime}}$ and $m_{d^{\prime}}-m_{u^{\prime}} \gg m_{e^{\prime}}$ so that the lightest baryon is $B_{u u u}^{\prime}$ and $B_{u u d}^{\prime}, B_{u d d}^{\prime}, B_{d d d}^{\prime}$ are unstable. The DM candidate is $(\mathrm{He})_{*}^{\prime}$ composed of (uuuee). (The star subscript indicates that the flavor structure of the nucleus differs from the corresponding SM case.) The constraints on $(\mathrm{He})_{*}^{\prime}$ dark matter are discussed later.

In regions where $m_{d^{\prime}}-m_{u^{\prime}} \sim m_{e^{\prime}}$, the other baryons, $B_{u u d}^{\prime}, B_{u d d}^{\prime}, B_{d d d}^{\prime}$, could be the lightest baryon, and $B_{u u u}^{\prime}, B_{u u d}^{\prime}, B_{u d d}^{\prime}, B_{d d d}^{\prime}, e^{\prime}$ may all be stable. The spectrum of 

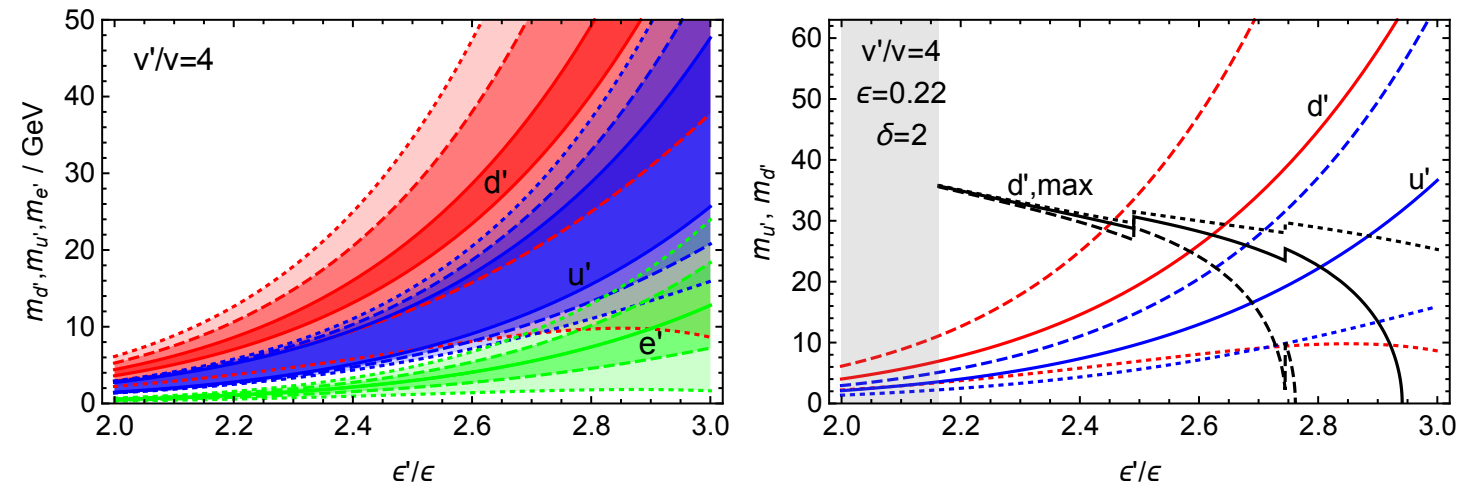

Figure 3. Left panel: the masses of $u^{\prime}, d^{\prime}$ and $e^{\prime}$, including uncertainties from the SM up and down quark Yukawa couplings. Solid, dashed and dotted lines show the cases with $\delta=0,1,2$ respectively. Right panel: red and blue lines show the central value and $\delta=2$ ranges of the $d^{\prime}$ and $u^{\prime}$ masses, without any SM Yukawa uncertainties. Black lines show the maximum $d^{\prime}$ mass allowed from the Higgs signal strength, showing the central and $\delta=2$ range as $u^{\prime}$ and $d^{\prime}$ masses are varied.
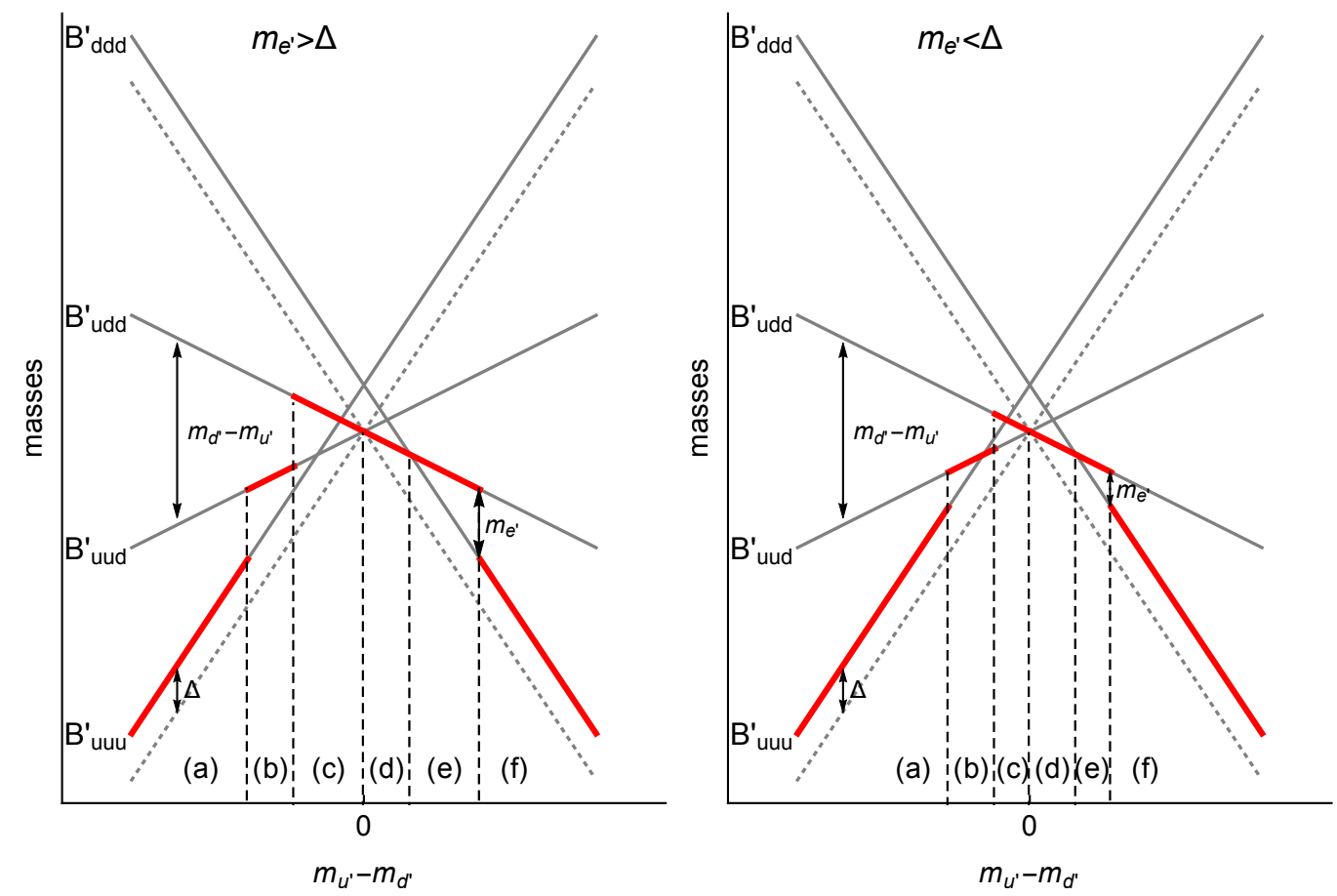

Figure 4. The mass spectrum of the mirror baryons as a function of $m_{u^{\prime}}-m_{d^{\prime}}$. The dotted lines show the masses of $B_{u u u}^{\prime}$ and $B_{d d d}^{\prime}$ ignoring the contribution from the mirror QCD dynamics to the mass difference between the lightest spin-3/2 baryons and the lightest spin- $1 / 2$ baryons, $\Delta$. The red lines show the mirror baryon of the dark matter candidate. 


\begin{tabular}{|c|c|c|c|c|}
\hline & $m_{e^{\prime}}+\Delta<\delta m_{d^{\prime} u^{\prime}}$ & $m_{e^{\prime}}<\delta m_{d^{\prime} u^{\prime}}<m_{e^{\prime}}+\Delta$ & $-\Delta-m_{e^{\prime}}<\delta m_{d^{\prime} u^{\prime}}<m_{e^{\prime}}$ & $\delta m_{d^{\prime} u^{\prime}}<-m_{e^{\prime}}-\Delta$ \\
\hline $\mathrm{DM}$ & $B_{u u u}^{\prime}+2 e^{\prime}$ & $B_{u u d}^{\prime}+e^{\prime}$ & $B_{u d d}^{\prime}$ & $B_{d d d}^{\prime}+e^{\prime}$ \\
\hline
\end{tabular}

Table 2. Ranges of $m_{d^{\prime}}-m_{u^{\prime}} \equiv \delta m_{d^{\prime} u^{\prime}}$ for the four Dark Matter candidates.

these baryons is sketched in figure 4, for $m_{e^{\prime}}>\Delta\left(m_{e^{\prime}}<\Delta\right)$ in the left (right) panel. In appendix $\mathrm{D}$ we show that, after freeze-out of the mirror weak interactions at a temperature of about $m_{e^{\prime}} / 18$, the baryon asymmetry is always carried by the lightest baryon, even if the heavier ones are stable.

Hence there are four DM candidates

$$
(\text { He })_{*}^{\prime}(\text { uuuee }), \quad H^{\prime}(\text { uude }), \quad n^{\prime}(u d d), \quad H_{*}^{\prime}(d d d \bar{e})
$$

Regions of parameter space leading to these four candidates are shown in figure 5 , separated by black dashed lines, with the predicted regions in the SU(5) model shown by dark (light) red shading for $\delta=1(2)$, with $\delta_{e}=0$. The $n^{\prime}$ candidate is particularly important since the others are atoms and are significantly constrained by limits on self-scattering and relic ionization, as described below. It is interesting and remarkable that the $n^{\prime}$ region of figure 5 is large, arising from a large region with $m_{d^{\prime}}-m_{u^{\prime}} \sim m_{e^{\prime}}$, while the $H^{\prime}$ and $H_{*}^{\prime}$ regions are smaller.

While weak interaction freeze-out puts the baryon asymmetry into the lightest baryon, when atomic states form the electron capture process, if kinematically allowed, ensures that

$$
(H e)_{*}^{\prime}(\text { uuuee }) \rightarrow H^{\prime}(\text { uude }), \quad H^{\prime}(\text { uude }) \rightarrow n^{\prime}(u d d) \quad H_{*}^{\prime}(d d d \bar{e}) \rightarrow n^{\prime}(u d d)
$$

so that the DM candidate is the lightest of $(H e)_{*}^{\prime}, H^{\prime}, n^{\prime}$ and $H_{*}^{\prime}$. It is the latter two processes that significantly enhance the $n^{\prime} \mathrm{DM}$ region. In figure 4 the red line tracks the baryon of the DM candidate, and jumps where electron capture occurs, so that the DM candidate does not necessarily contain the lightest baryon. The growth in the $n^{\prime} \mathrm{DM}$ region is particularly pronounced for large $m_{e^{\prime}}$. The resulting ranges of $m_{d^{\prime}}-m_{u^{\prime}}$ for each of the four DM candidates are shown in table 2.

\subsubsection{Direct detection via Higgs exchange}

Before investigating constraints and signals peculiar to each dark matter candidate, we discuss a signal universal to all the above candidates. These dark matter particles interact with SM nucleons through the exchange of the SM-like Higgs, $h$, and can be observed in direct detection experiments [5, 40-42]. The scattering cross section between a nucleon and a dark matter particle is given by [5]

$$
\sigma_{N, \mathrm{DM}}=\frac{0.028}{\pi} \frac{m_{\mathrm{DM}}^{2} m_{N}^{2}}{v^{\prime 4} m_{h}^{4}}\left(\frac{m_{N} m_{\mathrm{DM}}}{m_{N}+m_{\mathrm{DM}}}\right)^{2}
$$

where $m_{N}$ and $m_{\mathrm{DM}}$ are the masses of the nucleon and the DM, respectively. Here we assume that the mass of dark matter is dominated by mirror fermion masses. This cross section is shown in figure 6 . We also show constraints from the XENOT1T experiment (30days) [43], the expected sensitivities of XENON1T [44], LZ [45] and DARWIN [46] experiments, as well as the neutrino floor [47]. 

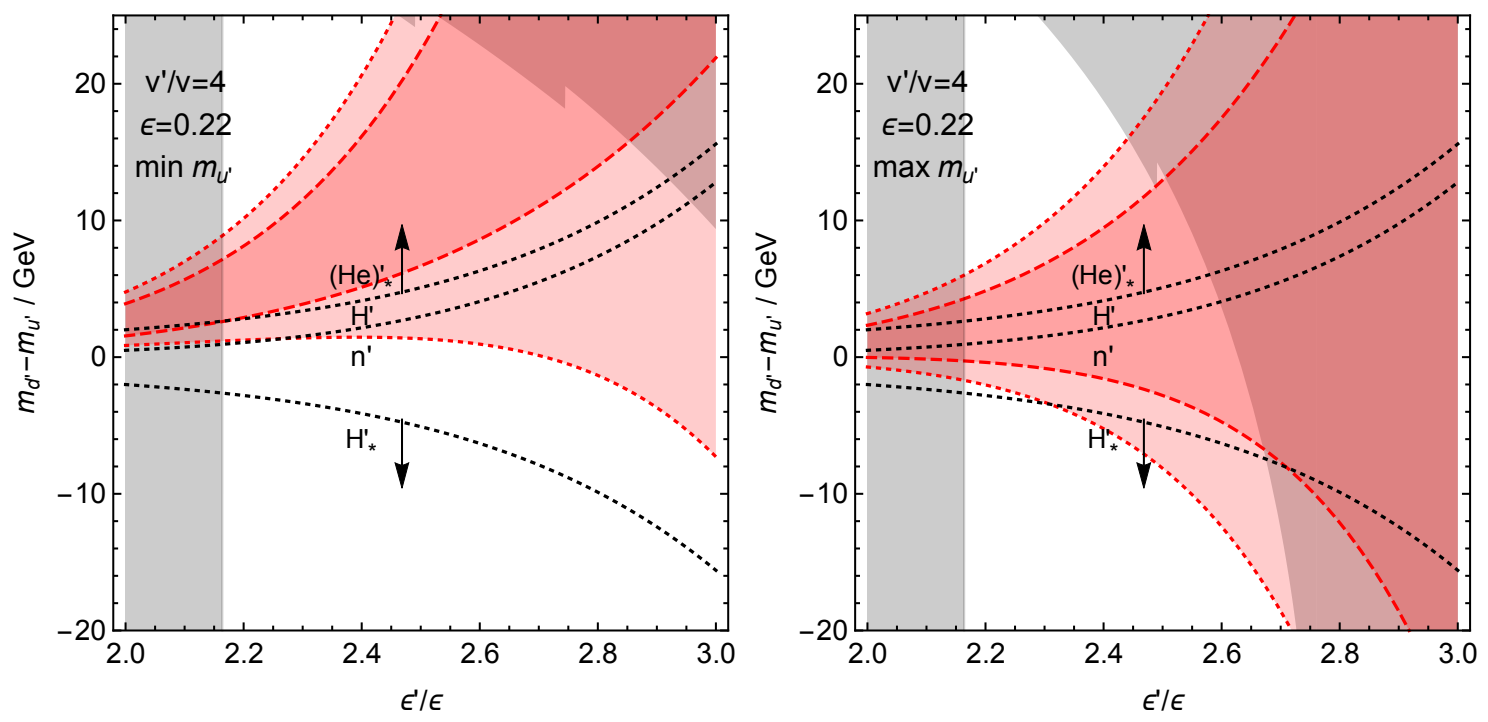

Figure 5. Dark (light) red shading gives the range of $m_{d^{\prime}}-m_{u^{\prime}}$ for $\delta=1(2)$. Black dashed lines separate regions where the DM candidate is $(H e)_{*}^{\prime}, H^{\prime}, n^{\prime}$ and $H_{*}^{\prime}$ with $\delta_{e}=0$. The left (right) panel is for minimal (maximal) $m_{u^{\prime}}$. Gray shaded regions are excluded by the Higgs signal strength. The position of the upper and lower black dotted lines are uncertain and are shown for $\Delta=T_{c}^{\prime}$.

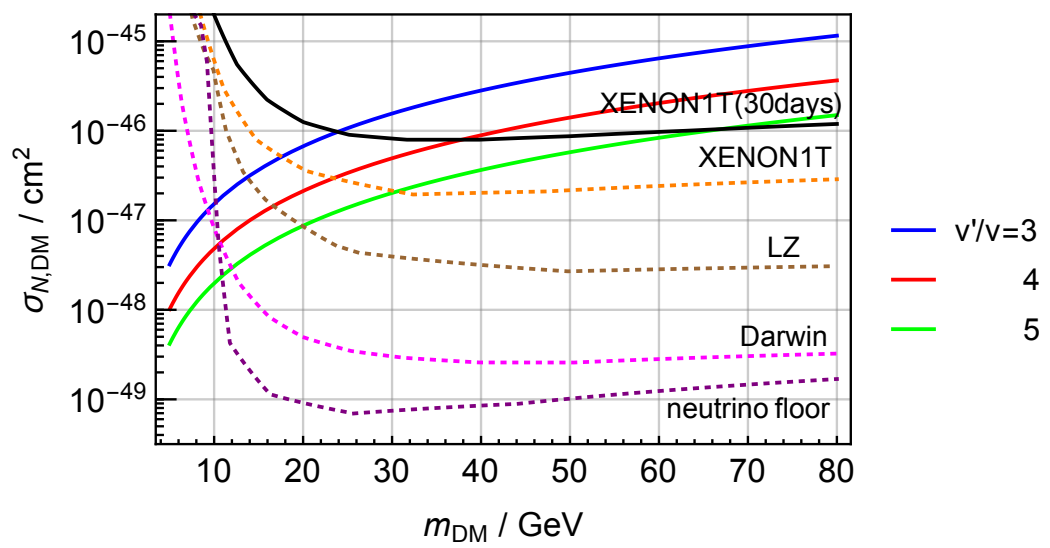

Figure 6. The scattering cross section between a dark matter particle and a SM nucleon as a function of the dark matter mass, which we assume is dominated by mirror fermion masses. The three full coloured lines correspond to $v^{\prime} / v=3,4,5$.

\subsubsection{Constraint on $(\mathrm{He})_{*}^{\prime}$ dark matter: region (a) of figure 4}

In Region (a) of figure 4, where $d^{\prime}$ is sufficiently heavy, the lightest baryon is $B_{u u u}^{\prime}$. Therefore the mirror matter asymmetry results in the asymmetric components of $B_{u u u}^{\prime}$ and $e^{\prime}$, which are stable cosmological relics. Once most of these combine into $(\mathrm{He})_{*}^{\prime}$, they may explain the observed dark matter in the universe.

We calculate the recombination of $(\mathrm{He})_{*}^{\prime}$, following the method described in [48], which calculates the recombination in the SM. We rescale recombination coefficients, etc, according to $m_{e^{\prime}} / m_{e}$. This is applicable as long as $m_{u^{\prime} u^{\prime} u^{\prime}} \gg m_{e^{\prime}}$. The temperature of mirror 


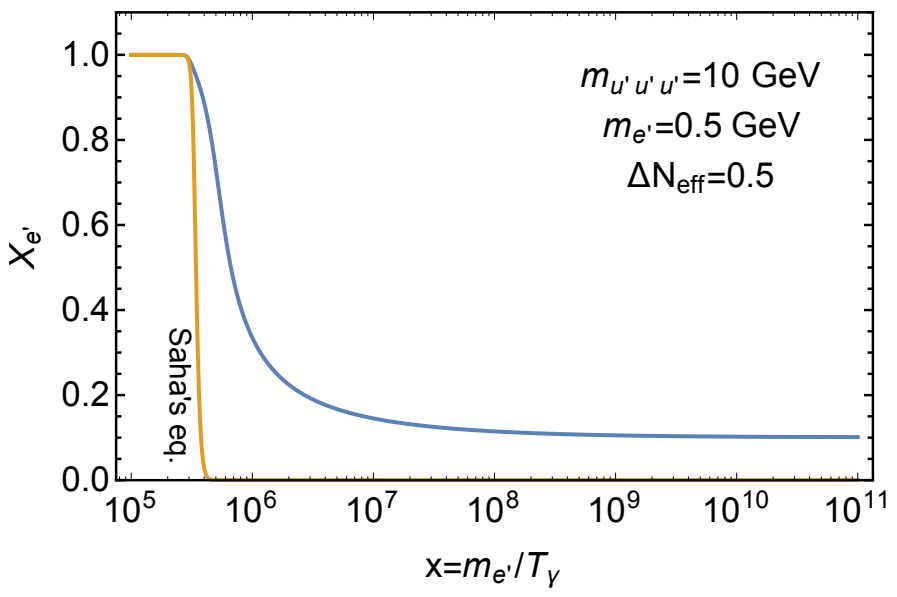

Figure 7. A sample evolution of the ionization fraction of $(\mathrm{He})_{*}^{\prime}$.

photons is determined via

$$
T_{\gamma^{\prime}} / T_{\gamma}=\left(\frac{7}{29} \Delta N_{\mathrm{eff}}\right)^{1 / 4}\left(\frac{4}{11}\right)^{1 / 3} \simeq 0.42\left(\frac{\Delta N_{\mathrm{eff}}}{0.5}\right)^{1 / 4}
$$

A sample evolution of the ionization fraction of the mirror electron, $X_{e^{\prime}}$, is shown in figure 7 . At low temperatures we find

$$
X_{e^{\prime}} \simeq 0.05\left(\frac{m_{u^{\prime} u^{\prime} u^{\prime}}}{10 \mathrm{GeV}}\right)^{0.8}\left(\frac{m_{e^{\prime}}}{0.23 \mathrm{GeV}}\right)^{0.8}\left(\frac{\Delta N_{\mathrm{eff}}}{0.5}\right)^{1 / 4}
$$

where we assume $m_{\mathrm{u}^{\prime} \mathbf{u}^{\prime} \mathbf{u}^{\prime}} / m_{e}^{\prime} \gg 1$. The sudden decoupling approximation from Saha's equation predicts $X_{e^{\prime}} \propto m_{u^{\prime} u^{\prime} u^{\prime}} m_{e^{\prime}}$, but, as it can be seen in figure 7, the approximation is far from perfect. Since the ionized components scatter with each other with a long-range force, their fraction is constrained by the possible change of the mass-to-luminosity ratio in the Bullet Cluster $[49,50], X_{e^{\prime}} \lesssim 0.3$.

The $(\mathrm{He})_{*}^{\prime}$ self-interaction cross section at low velocity is given by

$$
\frac{\sigma}{m_{\mathrm{DM}}} \simeq \frac{f\left(m_{u^{\prime} u^{\prime} u^{\prime}} / m_{e^{\prime}}\right)}{m_{e^{\prime}}^{2} \alpha^{2}} \frac{1}{m_{u^{\prime} u^{\prime} u^{\prime}}}=8.2 \mathrm{~cm}^{2} / \mathrm{g} \times \frac{10 \mathrm{GeV}}{m_{u^{\prime} u^{\prime} u^{\prime}}}\left(\frac{1 \mathrm{GeV}}{m_{e^{\prime}}}\right)^{2} \frac{f\left(m_{u^{\prime} u^{\prime} u^{\prime}} / m_{e^{\prime}}\right.}{20} .
$$

We evaluate the function $f$ by calculating the s-wave scattering cross section using the HFDHE2 potential [51]. The numerical value of $f\left(m_{u^{\prime} u^{\prime} u^{\prime}} / m_{e^{\prime}}\right)$ is given in figure 8. We adopt the constraint $\sigma / m_{\mathrm{DM}}<10 \mathrm{~cm}^{2} / \mathrm{g}$ [52]. The Bullet Cluster gives a stronger constraint on $\sigma / m_{\mathrm{DM}}$. However, the velocity of dark matter there is large, $v \sim 10^{-2} c$, so that the typical momentum exchanged between dark matter exceeds the inverse of the Bohr radius of $(\mathrm{He})_{*}^{\prime}$, giving a scattering cross section significantly suppressed relative to the low velocity one in eq. (3.15).

In the top left panel of figure 9, the shaded regions are excluded by the constraint on $\left(m_{e^{\prime}}, m_{u^{\prime} u^{\prime} u^{\prime}}\right)$ from the ionization fraction and the self-interaction. A portion of the 


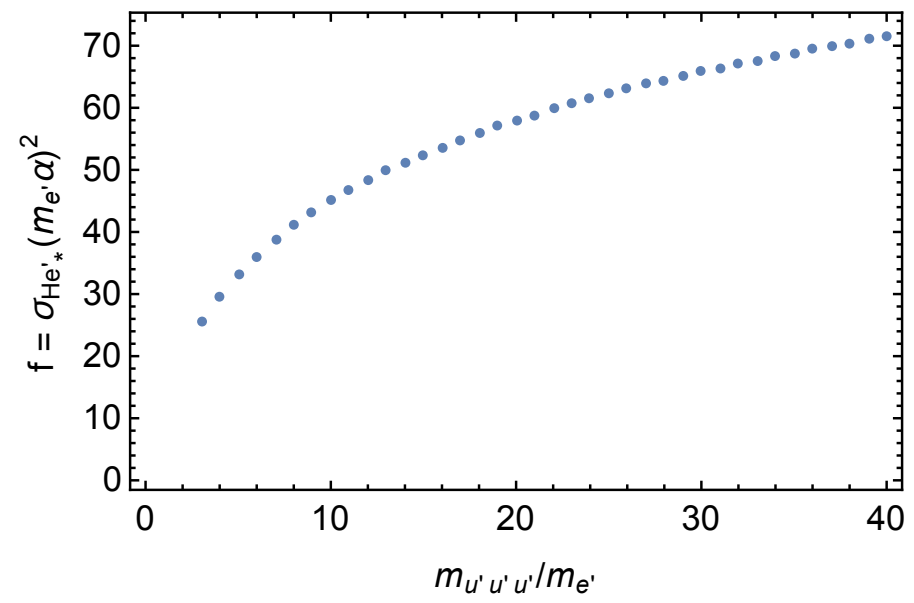

Figure 8. The normalized self-interaction cross section of $(H e)_{*}^{\prime}, f$, of eq. (3.15).

parameter space is allowed. Solid lines show the prediction of the $\mathrm{SU}(5)$-consistent FN model for $\left(m_{e^{\prime}}, m_{u^{\prime} u^{\prime} u^{\prime}}\right)$. The lines labeled " $\delta=0,1,2$ " show the range of the prediction with $\left|\delta_{f}\right|=0,1,2$, taking into account the $30 \%$ uncertainty of the Yukawa coupling of the up quark. We choose the signs of $\delta_{u, e}$ and the uncertainty of $y_{u}$ so that the upper (lower) two lines are located to the upper-left (lower-right). Here we neglect the difference between $m\left(B_{u^{\prime} u^{\prime} u^{\prime}}\right)$ and $m_{u^{\prime} u^{\prime} u^{\prime}}=3 m_{u^{\prime}}$. For small $m_{u}^{\prime}$ the contribution from the mirror QCD dynamics is non-negligible, and the solid lines would slightly rise. $\epsilon^{\prime} / \epsilon \lesssim 2.2$ is excluded by the measurement of the Higgs signal strength. It can be seen that $\epsilon^{\prime} / \epsilon=2.2-2.4$ predicts values of $\left(m_{e^{\prime}}, m_{u^{\prime} u^{\prime} u^{\prime}}\right)$ consistent with the constraints, and the mass of dark matter is in the range $(10-20) \mathrm{GeV}$. All of this range is currently allowed by data from XENON1T, but much of the upper range will be probed by XENON 1T, LZ and DARWIN, as shown by the dashed lines.

\subsubsection{Constraints on $H^{\prime} / H_{*}^{\prime}$ dark matter: regions (b) and (f) of figure 4}

In Region (f) where $u^{\prime}$ is sufficiently heavier than $d^{\prime}$, the lightest baryon is $B_{d d d}^{\prime}$, so that the mirror asymmetry is in the asymmetric components of $B_{d d d}^{\prime}$ and $e^{\prime}$. They may recombine into a neutral atom $H_{*}^{\prime}$ and explain the observed dark matter. The discussion here also applies to Region (b). There the lightest baryon is $B_{\text {uuu }}^{\prime}$, but, once the recombination $B_{u u u}^{\prime}+e^{\prime} \rightarrow H e_{*}^{\prime}$ happens, $H e_{*}^{\prime}$ decays into $B_{u u d}^{\prime}+\nu^{\prime}$, and the recombination $B_{u u d}^{\prime}+e^{\prime} \rightarrow H^{\prime}$ follows. The first recombination process is more efficient than the second one due to the larger charge of the nucleon, so that we may approximate the whole recombination process as that of $B_{u u d}^{\prime}+e^{\prime}$. We denote the mirror baryons of unit charge $\left(B_{u u d}^{\prime}\right.$ or $\left.B_{d d d}^{\prime}\right)$ as $B^{\prime+}$.

We calculate the recombination of the mirror baryon and mirror electron following [48]. We find the ionization fraction,

$$
X_{e^{\prime}} \simeq 0.05\left(\frac{m_{B^{\prime+}}+m_{e^{\prime}}}{10 \mathrm{GeV}}\right)^{0.9}\left(\frac{m_{\mathrm{red}, e^{\prime} B^{\prime}}}{0.94 \mathrm{GeV}}\right)^{0.9}\left(\frac{\Delta N_{\mathrm{eff}}}{0.5}\right)^{1 / 4},
$$



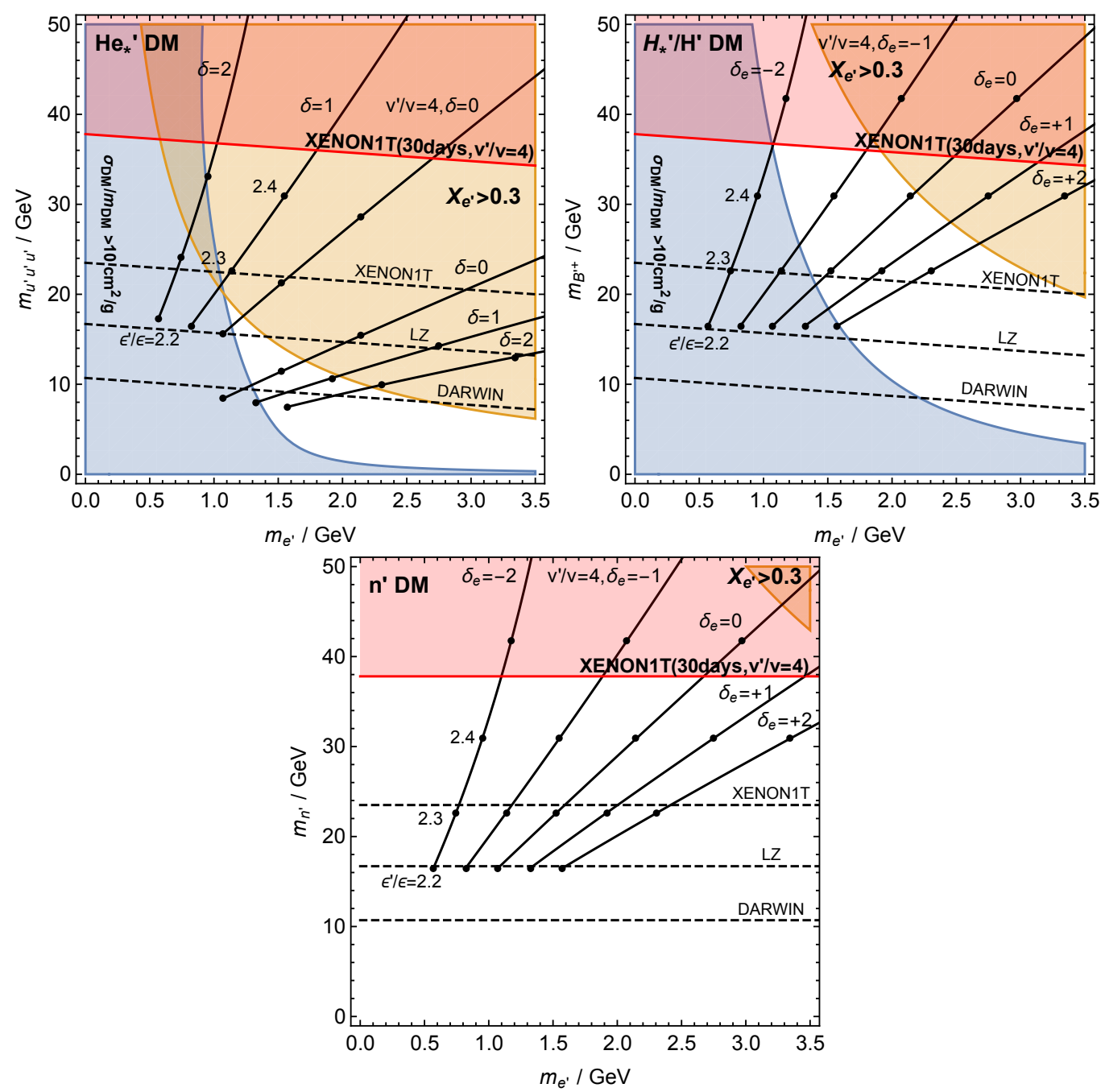

Figure 9. Constraints on the masses of $B_{u^{\prime} u^{\prime} u^{\prime}}$ (top left panel) or $B_{d^{\prime} d^{\prime} d^{\prime}} / B_{u^{\prime} u^{\prime} d^{\prime}}$ (top right panel) and $e^{\prime}$ from self-interactions of mirror atoms, the mirror ionization fraction and direct detection. The bottom panel assumes that the mass of $B_{u^{\prime} d^{\prime} d^{\prime}}=n^{\prime}$ is below $m_{B^{\prime+}}+m_{e}^{\prime}$ and the mirror electron capture occurs inside the mirror atom. Solid curves show predictions of the SU(5)-compatible model for a range of the uncertainties, as described in the text. Dashed curves give expected reaches of future direct detection experiments.

where $m_{\mathrm{red}, e^{\prime} B^{\prime}}$ is the reduced mass of the mirror electron-baryon system. For fixed mirror baryon and electron masses, the ionization fraction of $H^{\prime} / H_{*}^{\prime}$ is smaller than that of $(\mathrm{He})_{*}^{\prime}$, since the recombination cross section is larger for $H^{\prime} / H_{*}^{\prime}$.

For $m_{B^{\prime}} / m_{e^{\prime}}=O(1-10)$, the $H^{\prime} / H_{*}^{\prime}$ self-interaction cross section is given by [53]

$$
\frac{\sigma}{m_{\mathrm{DM}}} \simeq \frac{100}{m_{e^{\prime}}^{2} \alpha^{2}} \frac{1}{m_{\mathrm{DM}}} \simeq 5.1 \mathrm{~cm}^{2} / \mathrm{g} \times \frac{20 \mathrm{GeV}}{m_{B^{\prime}}}\left(\frac{2 \mathrm{GeV}}{m_{e^{\prime}}}\right)^{2}
$$

In the top right panel of figure 9 , we show the constraints on $\left(m_{e^{\prime}}, m_{B^{\prime+}}\right)$ from the ionization fraction and the self-interaction. In order for $H^{\prime}\left(H_{*}^{\prime}\right)$ to be dark matter, $m_{u^{\prime}}$ 
must be similar to (larger than) $m_{d^{\prime}}$. The right bottom panel of figure 5 shows that this is possible if $m_{u^{\prime}}\left(m_{d^{\prime}}\right)$ is larger (smaller) than its central value. The figure also shows that for $\left|\delta_{f}\right|<1, m_{d^{\prime}}$ is not much smaller than $m_{u^{\prime}}$. Based on these observations, in the right panel of figure 9 we show predictions for $\left(m_{e^{\prime}}, 3 m_{d^{\prime}}\right)$ by solid lines, fixing $\delta_{u}=+1$ and the SM up Yukawa coupling larger than its central value by $30 \%$. The various solid lines show that much of the allowed space is possible with $\left|\delta_{e}\right|<2$. Hence, with $\epsilon^{\prime} / \epsilon=2.2-2.5$ the prediction for $\left(m_{e^{\prime}}, m_{B^{\prime+}}\right)$ is consistent with the constraints, and the mass of dark matter is in the range $(20-50) \mathrm{GeV}$. Xenon1T, LZ and DARWIN will probe all of this range.

\subsubsection{Constraints on $n^{\prime}$ dark matter: regions (c), (d) and (e) of figure 4}

In Region (d) the lightest baryon is $B_{u d d}^{\prime}=n^{\prime}$, so that the mirror asymmetry is in the asymmetric component of $n^{\prime}$. There is no constraint from the ionization fraction or from the self-interaction cross section.

In Regions (c) and (e) the lightest baryon is not $n^{\prime}$ but a charged mirror baryon. However, once recombination happens, the mirror atom decays into $n^{\prime}+\nu$, yielding $n^{\prime}$ as a stable particle. Still, the recombination may not be complete and there would be a constraint from the ionization fraction. In Region (e) the recombination process is $B_{d d d}^{\prime}+$ $\bar{e}^{\prime} \rightarrow H_{*}^{\prime}$, while in the right part of Region (c) it is $B_{u u d}^{\prime}+e^{\prime} \rightarrow H^{\prime}$. In the left part of Region (c) the recombination proceeds via $B_{u u u}^{\prime}+e^{\prime} \rightarrow H e_{*}^{\prime}, H e_{*}^{\prime} \rightarrow B_{u u d}^{\prime}+\nu^{\prime}$, and $B_{u u d}^{\prime}+e^{\prime} \rightarrow H^{\prime}$. The first and the second reaction is more efficient than the last one, so that we may approximate the whole process as $B_{u u d}^{\prime}+e \rightarrow H^{\prime}$. Thus in Regions (c) and (e) the recombination process is described as that of a mirror baryon with unit charge $\left(B^{\prime}\right)$ and $e^{\prime}$.

In appendix E we calculate the ionization fraction with the inclusion of electron capture. We find that the ionization fraction is well-fitted by the following formula,

$$
X_{e^{\prime}} \simeq 0.05\left(\frac{m_{n^{\prime}}}{10 \mathrm{GeV}} \frac{m_{\mathrm{red}, e^{\prime} B^{\prime}}}{1.6 \mathrm{GeV}}\right)^{0.8}\left(\frac{\Delta N_{\mathrm{eff}}}{0.5}\right)^{1 / 4} .
$$

In the bottom panel of figure 9 , the corresponding constraint on $\left(m_{e^{\prime}}, m_{n^{\prime}}\right)$ is shown. The constraint is weaker than that on $H^{\prime} / H_{*}^{\prime}$ dark matter, as the electron capture removes the mirror atom from the thermal bath, inhibiting the inverse process $H^{\prime} / H_{*}^{\prime}+\gamma^{\prime} \rightarrow B^{\prime+}+e^{\prime}$. The solid lines are the same as those in the top right panel, and show that $\epsilon^{\prime} / \epsilon<2.4$ is allowed with $\left|\delta_{e}\right|<2$. The mass of dark matter is in the range $(20-60) \mathrm{GeV}$. Part of the parameter region is excluded by XENON1T. Xenon1T, LZ and DARWIN will probe all of this range.

\subsubsection{Mirror and SM matter asymmetries}

As we have seen, in viable parameter regions the mass of dark matter is $O(10) \mathrm{GeV}$. Hence the observed dark matter abundance is explained by a mirror matter asymmetry of the same order as the SM matter asymmetry. A difference of $O(1)$ in the asymmetries may arise in some scenarios of baryogenesis. For example, if the baryon asymmetry is created by the Affleck-Dine mechanism [54, 55], an $O(1)$ difference is expected from the difference of the initial mis-alignment in the angular direction of the scalar field responsible for baryogenesis. 
A dark matter mass of $m_{p} \cdot \Omega_{\mathrm{DM}} / \Omega_{\mathrm{b}} \approx 5 \mathrm{GeV}$ is close to being allowed for $H e_{*}^{\prime}$ dark matter. This would be consistent with equal matter asymmetries in the standard and in the mirror sectors.

\subsubsection{Possibility of mirror nucleosynthesis}

Mirror baryons collide with each other and may form bound states, namely mirror nuclei [32]. Formation of nuclei of generic composite dark matter is discussed in [56, 57].

In our case first we argue that mirror nuclei composed of more than two baryons are unlikely to be formed. In most of the parameter space the mass difference between the lightest mirror baryon and the next to lightest one is much larger than $m_{e^{\prime}} / 18$, so that almost all of the mirror baryon number is stored in the lightest mirror baryons. Therefore, in order for the lightest mirror baryon to form a bound state with more than two baryons, a non-zero angular momentum is required due to Fermi statistics. This leads to a positive energy of order $1 /\left(m_{B^{\prime}} r^{2}\right)$, where $r$ is the radius of the bound state. We expect that $r^{-1}$ is as large as the mass of the mediator of the mirror strong force. In the parameter space of interest $m_{d^{\prime}, u^{\prime}}>T_{c}^{\prime}$, so $1 /\left(m_{B^{\prime}} r^{2}\right)=O\left(m_{u^{\prime}, d^{\prime}}\right)$. On the other hand the possible binding energy would be at most $O\left(T_{c}^{\prime}\right)<O\left(m_{d^{\prime} \cdot u^{\prime}}\right)$. Thus we expect that mirror nuclei composed of more than two lightest mirror baryons are unbound.

There could be a mirror nucleus composed of two lightest mirror baryons. A lattice QCD calculation with a quark mass larger than normal seems to make space for di-neutron and di-proton states $[58,59]$. Although it is not clear if a mirror di-neutron and di-proton exist for our mirror quark masses, or mirror di- $B_{u u u}^{\prime}$ and di- $B_{d d d}^{\prime}$ exist for any mirror quark mass, let us suppose that those states are stable and discuss the phenomenological consequence. To verify this assumption, a dedicated lattice calculation is needed.

Once the temperature drops below the binding energy, almost all of the lightest mirror baryons in figure 4 are combined into di-baryon states. In Region (a), a mirror baryon with charge 4 is formed. The recombination as well as the self-scattering cross section is affected, in a way that we do not pursue further in this paper. In Region (b), mirror di-protons are formed via the formation of di-baryons and the mirror electron capture. The constraint on $H e_{*}^{\prime}$ is applicable but with twice larger baryon mass. There is no viable parameter space for the SU(5) model. In Regions (c), (d) and (e), mirror di-neutrons are formed. The constraint on $n^{\prime}$ is again applicable with twice larger baryon mass. All parameter region of the SU(5) model with $v^{\prime} / v<4$ can be probed by the XENON1T. In Region (f) mirror di- $B_{d d d}^{\prime}$ are formed. The constraint on $H e_{*}^{\prime}$ is applicable but with twice larger baryon mass.

\subsection{Dark radiation}

In the early universe with a sufficiently large temperature the SM particles and their mirror partners interact with each other and have the same temperature. Below some temperature $T_{d}$ the interaction becomes inefficient and they evolve independently. Mirror particles eventually decay/annihilate into mirror photons and neutrinos, which are observed as dark radiation. The abundance of the dark radiation, traditionally expressed as the excess of 
the effective number of neutrinos from the SM prediction, is

$$
\Delta N_{\mathrm{eff}}=\frac{4}{7} g_{r}^{\prime} \times\left(\frac{10.75}{g\left(T_{d}\right)}\right)^{4 / 3} \times\left(\frac{g^{\prime}\left(T_{d}\right)}{g_{r}^{\prime}}\right)^{4 / 3},
$$

where $g(T)$ and $g^{\prime}(T)$ are the effective entropy degrees of freedom (d.o.f) of the SM particles and the mirror particles at temperature $T$, respectively. The second factor in the r.h.s. of eq. (3.19) expresses the heating of the SM neutrinos, whereas the third factor expresses the heating of the dark radiation. $g_{r}^{\prime}$ is the d.o.f. of the radiation component of the mirror sector. In the minimal model where the mirror neutrinos are nearly massless, $g_{r}^{\prime}=29 / 4$. We extract the d.o.f. of the SM particles $g(T)$ from [60].

\subsubsection{Generic decoupling temperature}

In this subsection we treat $T_{d}$ as a free parameter. If $T_{d}>T_{c}^{\prime}$, the mirror gluons give a large contribution to $g^{\prime}\left(T_{d}\right)$, and $\Delta N_{\text {eff }}$ is larger than the constraint from the Planck satellite, $\Delta N_{\text {eff }}<0.65(2 \sigma)$. We only consider the case with $T_{d}<T_{c}^{\prime}$, and neglect the contribution from the mirror gluons to $g^{\prime}\left(T_{d}\right)$.

The contributions of the mirror photons, neutrinos and leptons to $g^{\prime}\left(T_{d}\right)$ are readily estimated using the ideal gas approximation. The mirror quarks, on the other hand, cannot be treated as an ideal gas, especially for $T_{d}<T_{c}^{\prime}$, where the dynamics of the mirror quarks is better described as a gas of mirror hadrons. Figure 1 shows that among mirror hadrons, the ones composed of mirror up quarks are the most important ones. We estimate the contribution from the mirror QCD sector, treating the hadron gas as an ideal gas composed of mirror $\sigma(J=0, C P=++), \eta^{\prime}(J=0, C P=+-)$ and $\omega(J=1, C P=--)$, with their masses given by

$$
\begin{aligned}
& m_{\sigma}^{\prime 2}=\left(2 m_{u^{\prime}}\right)^{2}+\left(1.5 T_{c}^{\prime}\right)^{2} \\
& m_{\eta^{\prime}}^{\prime 2}=\left(2 m_{u^{\prime}}\right)^{2}+\left(3 T_{c}^{\prime}\right)^{2}, \\
& m_{\omega^{\prime}}^{\prime 2}=\left(2 m_{u^{\prime}}\right)^{2}+\left(4 T_{c}^{\prime}\right)^{2} .
\end{aligned}
$$

The contribution proportional to $T_{c}^{\prime 2}$ is inferred from the Standard Model QCD spectrum.

In figure 10, we show the prediction of $\Delta N_{\text {eff }}$ as a function of $T_{d}$ with fixed $m_{e^{\prime}}, m_{u^{\prime}}$ and $T_{c^{\prime}}$, neglecting the contributions from the other mirror fermions. The brown, red and green lines show the contribution from $\gamma^{\prime} \nu^{\prime}, \gamma^{\prime} \nu^{\prime} e^{\prime}$ and $\gamma^{\prime} \nu^{\prime} e^{\prime} \sigma^{\prime} \eta^{\prime \prime} \omega^{\prime}$, respectively. These figures show that $\Delta N_{\text {eff }}$ is dominated by the contribution from $\gamma^{\prime}, \nu^{\prime}$ and $e^{\prime}$. For comparison, we also show $\Delta N_{\text {eff }}$ calculated using the quark picture with the ideal gas approximation by a blue line: confinement suppresses the abundance of dark radiation.

In figure 11, we show the prediction of $\Delta N_{\text {eff }}$ as a function of $\epsilon^{\prime} / \epsilon$ and $T_{d}$. Here we choose the sign and the value of each $\delta_{f}$ so the $\mu$ becomes as large as possible, expect for $\delta_{e}$, for which we take $\delta_{e}=\delta$ to suppress $\Delta N_{\text {eff }}$. The red line shows the mirror QCD phase transition temperature $T_{c}^{\prime}$. Above this line the contribution from mirror gluons makes $\Delta N_{\text {eff }}$ unacceptably large. Blue shaded regions are excluded due to too small $\mu$, as discussed in section 3.2. The amount of the dark radiation is typically $\Delta N_{\text {eff }}=0.3-0.6$. 

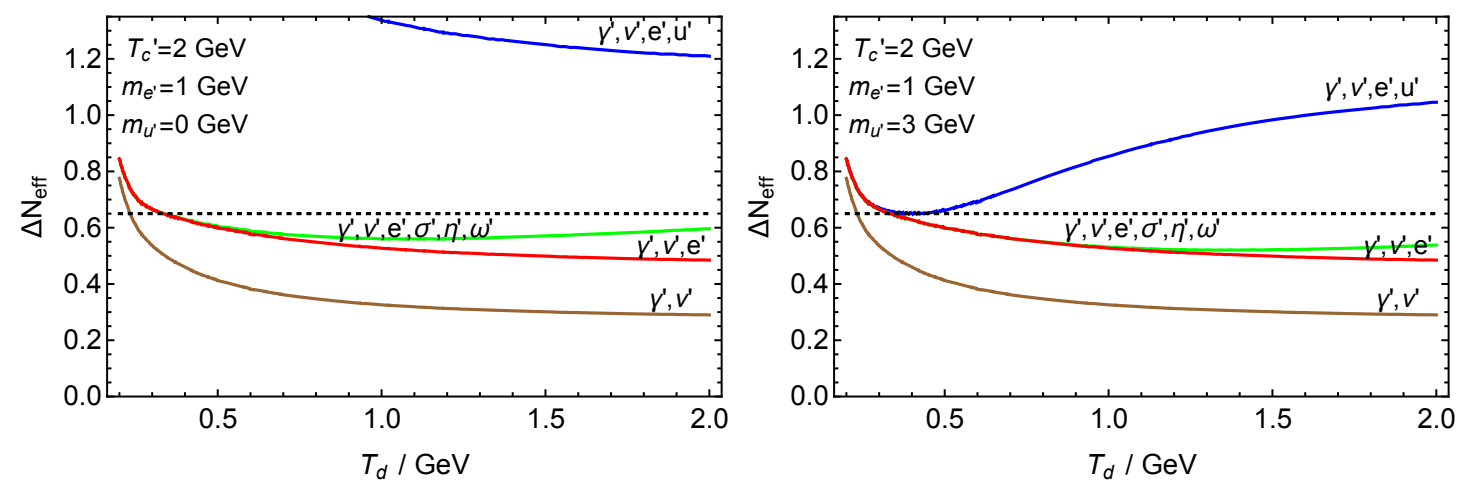

Figure 10. The dark radiation abundance predicted as a function of the decoupling temperature.
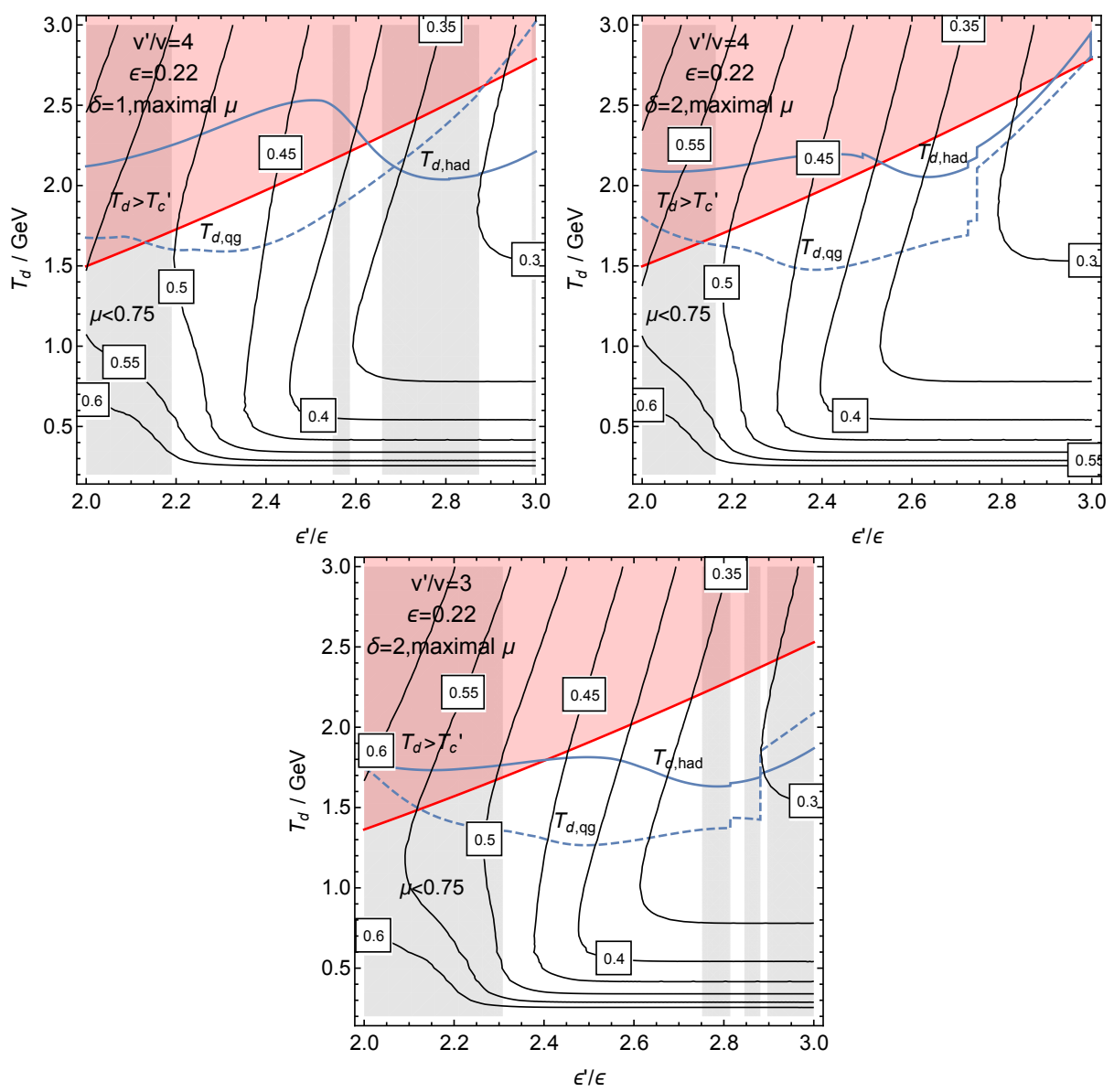

Figure 11. The predicted dark radiation abundance expressed as $\Delta N_{\text {eff }}$. We choose the mass spectrum of mirror fermions to minimize the invisible decay of the Higgs except for the mirror electron. In the red shaded region $T_{d}>T_{c}^{\prime}$ and the abundance of the dark radiation is too large. The solid and dashed blue lines show the decoupling temperature via the Higgs exchange in the hadron $\left(T_{d, h a d}\right)$ and the quark-gluon picture $\left(T_{d, q g}\right)$, respectively. For the quark-gluon picture the decoupling temperature is mainly determined by the annihilation of mirror quarks, while for the hadron picture it is mainly determined by the decay of mirror glueballs. 


\subsubsection{Decoupling temperature from Higgs exchange}

In this subsection we estimate the decoupling temperature determined by the Higgs exchange between the SM particles and the mirror partners. The interaction rate between the mirror leptons and the Standard fermions is readily estimated using the ideal gas picture, following [5]. The scattering cross section between a mirror fermion $f^{\prime}$ and a SM fermion $f$ is given by

$$
\sigma v_{\text {rel }}\left(f f^{\prime} \rightarrow f f^{\prime}\right)=\frac{1}{8 \pi}\left(\frac{m_{f}}{v}\right)^{2}\left(\frac{v m_{f^{\prime}}}{v^{\prime 2}}\right)^{2} \frac{m_{f} m_{f^{\prime}}}{m_{f}+m_{f^{\prime}}} \frac{p_{\mathrm{cm}}}{m_{h}^{4}},
$$

where we take a non-relativistic limit. Here $p_{\mathrm{cm}}$ is the momentum of the fermion in the center of mass frame. In the thermal bath, it has a typical size

$$
p_{\mathrm{cm}}^{2}=\frac{4 T\left(m_{f}+m_{f^{\prime}}+\sqrt{m_{f} m_{f^{\prime}}}\right)}{3\left(2+m_{f} / m_{f^{\prime}}+m_{f^{\prime}} / m_{f}\right)} .
$$

The annihilation cross section of a pair of $f^{\prime}$ into a pair of $f$ is given by

$$
\sigma\left(f^{\prime} \bar{f}^{\prime} \rightarrow f \bar{f}\right) v_{\mathrm{rel}}=\frac{N_{f}}{4 \pi}\left(\frac{m_{f}}{v}\right)^{2}\left(\frac{v m_{f^{\prime}}}{v^{\prime 2}}\right)^{2} \frac{\left(m_{f^{\prime}}^{2}-m_{f}^{2}\right)^{3 / 2}}{m_{f^{\prime}}^{3} m_{h}^{4}} p_{f^{\prime}}^{2} .
$$

Here $p_{f^{\prime}}$ is the momentum of $f^{\prime}$ in the center of mass frame. In the thermal bath, it is as large as $p_{f^{\prime}}^{2} \simeq 3 m_{f^{\prime}} T / 2 . \quad N_{f}$ is the multiplicity of the Dirac fermion $f$ : for one lepton (quark) $N_{f}=1(3)$. The transfer rate of the energy density of mirror particles into SM particles is then given by

$$
\begin{aligned}
\left.\frac{\mathrm{d}}{\mathrm{d} t} \rho^{\prime}\right|_{f^{\prime}}= & \sum_{f}\left(4 N_{f} n_{F}\left(m_{f}, T\right)\right)\left(4 N_{f^{\prime}} n_{F}\left(m_{f^{\prime}}, T\right)\right) \sigma v_{\mathrm{rel}}\left(f f^{\prime} \rightarrow f f^{\prime}\right) \times \Delta E \\
& +\sum_{f} N_{f^{\prime}} 4 n_{F}\left(m_{f^{\prime}}, T\right)^{2} \sigma v_{\mathrm{rel}}\left(f^{\prime} \bar{f}^{\prime} \rightarrow f \bar{f}\right) \times 2 m_{f^{\prime}},
\end{aligned}
$$

where $n_{F}(m, T)$ is the number density of a fermion of mass $m$ in the thermal bath at temperature $T$, and $\Delta E \simeq T$ is a typical energy transfer by the scattering $f f^{\prime} \rightarrow f f^{\prime}$.

The scattering with mirror QCD charged particles requires a dedicated treatment. We use in succession quark and hadron pictures with an ideal gas approximation to calculate the energy transfer rate.

Let us first treat mirror QCD charged particles as an ideal gas of mirror quarks and gluons. The scattering cross section between a mirror fermion $f^{\prime}$ and a SM fermion $f$ is given by eq. (3.21). The annihilation cross section of a pair of mirror quarks $q^{\prime}$ into a pair of $f$ is given by

$$
\begin{aligned}
\sigma\left(q^{\prime} \bar{q}^{\prime} \rightarrow f \bar{f}\right) v_{\text {rel }} & \simeq \frac{N_{f}}{4 \pi}\left(\frac{m_{f}}{v}\right)^{2}\left(\frac{v m_{f^{\prime}}}{v^{\prime 2}}\right)^{2} \frac{\left(m_{f^{\prime}}^{2}-m_{f}^{2}\right)^{3 / 2}}{m_{f^{\prime}}^{3} m_{h}^{4}} p_{f^{\prime}}^{2} \times \frac{2 \pi x}{1-e^{-2 \pi x}}\left(1+x^{2}\right), \\
x & =\frac{4}{3} \frac{\alpha_{s}^{\prime}}{v_{\text {rel }}}
\end{aligned}
$$


where we have included the Sommerfeld effect [61] for a $p$-wave annihilation [62]. The fine structure constant should be evaluated at the scale $\mu \simeq 4 / 3 m_{q^{\prime}} \alpha_{s}^{\prime} / 2$ [63], so we solve the consistency condition

$$
\frac{2}{3} m_{q^{\prime}} \alpha_{s}^{\prime}(\mu)=\mu
$$

to determine the appropriate scale. We put $v_{\text {rel }}=\sqrt{T / m_{q^{\prime}}}$ to estimate the Sommerfeld enhancement factor. The contribution of a mirror quark $q^{\prime}$ to the energy transfer rate is given by eq. (3.24).

The mirror gluons also couple to the SM Higgs,

$$
\mathcal{L}=\frac{v}{v^{\prime}} \frac{h}{\sqrt{2} v^{\prime}} \frac{\alpha_{s}^{\prime}}{12 \pi} \sum_{q^{\prime}}\left(1+\frac{11}{4 \pi} \alpha_{s}^{\prime}\left(m_{q^{\prime}}\right)\right) G_{\mu \nu}^{a^{\prime}} G^{\mu \nu a^{\prime}} \simeq \frac{v}{v^{\prime}} \frac{h}{\sqrt{2} v^{\prime}} \frac{\alpha_{s}^{\prime}}{2 \pi} G_{\mu \nu}^{a^{\prime}} G^{\mu \nu a^{\prime}} .
$$

The annihilation cross section of a pair of mirror gluons into a pair of SM fermions $f$ is

$$
\sigma\left(g^{\prime} g^{\prime} \rightarrow f \bar{f}\right) v_{\mathrm{rel}}=\frac{2 N_{f}}{\pi}\left(\frac{v}{v^{\prime}}\right)^{2}\left(\frac{\alpha_{s}^{\prime}}{2 \pi}\right)^{2}\left(\frac{m_{f}}{v}\right)^{2} \frac{1}{v^{\prime 2}}\left(\frac{p_{\mathrm{cm}}}{m_{h}}\right)^{4}\left(1-\frac{m_{f}^{2}}{p_{\mathrm{cm}}^{2}}\right)^{3 / 2},
$$

while the scattering cross section is

$$
\sigma\left(g^{\prime} f \rightarrow g^{\prime} f\right)=\frac{4}{3 \pi}\left(\frac{v}{v^{\prime}}\right)^{2}\left(\frac{\alpha_{s}^{\prime}}{2 \pi}\right)^{2}\left(\frac{m_{f}}{v}\right)^{2} \frac{1}{v^{\prime 2}}\left(\frac{p_{\mathrm{cm}}}{m_{h}}\right)^{4},
$$

where we take the non-relativistic limit for $f$. Due to the absence of the Sommerfeld effect, however, the energy transfer from mirror gluons is negligible in comparison with that from mirror quarks.

We define the decoupling temperature by $\left(\mathrm{d} \rho^{\prime} / \mathrm{d} t\right) / \rho^{\prime}=H$, where $H$ is the $T$-dependent expansion rate of the universe. In figure 11 , we show the decoupling temperature $T_{d, q g}$ determined by the Higgs exchange with the quark picture by dotted lines. We find that $T_{d, q g}$ can be lower than $T_{c}^{\prime}$. The decoupling temperature is dominantly determined by the annihilation of mirror quarks. We note, however, that this does not mean that the actual decoupling temperature $T_{d}$ can be below $T_{c}^{\prime}$. As the temperature drops and becomes close to $T_{c}^{\prime}$, the ideal gas approximation of mirror quarks is not straightforwardly applicable, and is expected to break down for $T_{d}<T_{c}^{\prime}$. Our estimate at least shows, however, that the decoupling temperature is close to $T_{c}^{\prime}$.

Let us next treat the mirror QCD charged particles as an ideal gas of mirror hadrons. We include the scattering and the annihilation of mirror glueballs. A spin-0 glueball with $C P=++$ mixes with the SM Higgs and decays into SM fermions. A result of a lattice calculation is available for the lightest one, $S_{0^{++}}^{\prime}$. Using the lattice calculation for the relevant matrix element and for the glueball mass [64],

$$
<0\left|g_{s}^{\prime 2} G_{\mu \nu}^{a^{\prime}} G^{\mu \nu a^{\prime}}\right| S_{0^{++}}>\simeq 2.7 m_{S_{0^{++}}^{\prime}}^{3}, \quad m_{S_{0^{++}}^{\prime}} \simeq 5.3 T_{c}^{\prime},
$$

the decay rate of $S_{0^{++}}^{\prime}$ into a pair of Standard Model fermion $f$ is given by

$$
\Gamma\left(S_{0^{++}}^{\prime} \rightarrow f \bar{f}\right)=\frac{1}{32 \pi}\left(\frac{v}{v^{\prime}}\right)^{2}\left(\frac{1}{8 \pi^{2}}\right)^{2}\left(\frac{m_{f}}{v}\right)^{2} \frac{1}{v^{\prime 2}} \frac{2.7^{2} m_{S_{0^{++}}^{\prime}}^{7}}{m_{h}^{4}}\left(1-\frac{4 m_{f}^{2}}{m_{S_{0^{+}+}^{\prime}}^{2}}\right)^{3 / 2}
$$


The scattering cross section of a mirror glueball $S_{i}^{\prime}$ can be estimated by the trace anomaly,

$$
<S_{i}^{\prime}\left|\frac{11}{32 \pi^{2}} g_{s}^{2^{\prime}} G_{\mu \nu}^{a^{\prime}} G^{\mu \nu a^{\prime}}\right| S_{i}^{\prime}>=2 m_{S_{i}^{\prime}}^{2}
$$

where we assume that the mass of the mirror glueball is not affected by the masses of mirror fermions, which is the case for sufficiently large mirror fermion masses and/or large $N_{c}$. The scattering cross section between a mirror glueball $S_{i}^{\prime}$ and $f$ is given by

$$
\sigma v_{\mathrm{rel}}\left(f S_{i}^{\prime} \rightarrow f S_{i}^{\prime}\right)=\frac{1}{8 \pi}\left(\frac{4}{11}\right)^{2}\left(\frac{m_{f}}{v}\right)^{2}\left(\frac{v m_{S_{i}^{\prime}}}{v^{\prime 2}}\right)^{2} \frac{m_{f} m_{S_{i}^{\prime}}}{m_{f}+m_{S_{i}^{\prime}}} \frac{p_{\mathrm{cm}}}{m_{h}^{4}} .
$$

We take into account the scattering with mirror glueballs of spin $S_{S_{i}}=0,1,2$ and $C P=$ ,,,+++--+-- , whose masses are estimated in [65]. The contribution of the mirror glueballs to the energy transfer rate is given by

$$
\begin{aligned}
\left.\frac{\mathrm{d}}{\mathrm{d} t} \rho^{\prime}\right|_{S}= & \sum_{f, i}\left(4 N_{f} n_{F}\left(m_{f}, T\right)\right)\left(\left(2 S_{S_{i}}+1\right) n_{B}\left(m_{S_{i}^{\prime}}, T\right)\right) \sigma v_{\mathrm{rel}}\left(f S_{i}^{\prime} \rightarrow f S_{i}^{\prime}\right) \times \Delta E \\
& +\sum_{f} n_{B}\left(m_{S_{0^{++}}}, T\right) \Gamma\left(S_{0^{++}} \rightarrow f \bar{f}\right) \times m_{S_{0^{++}}},
\end{aligned}
$$

where $n_{B}(m, T)$ is the number density of a boson of mass $m$ in the thermal bath at temperature $T$.

We also include the annihilation and the scattering of mirror quarkonia. The decay rate of a mirror quarkonium with spin-0 and $C P=++, \chi_{q^{\prime}}$, into a pair of SM fermions is approximately given by

$$
\left.\Gamma\left(\chi_{q^{\prime}} \rightarrow f \bar{f}\right) \simeq \sigma\left(q^{\prime} \bar{q}^{\prime} \rightarrow f \bar{f}\right) v_{\mathrm{rel}}\right|_{p_{q^{\prime}}=m_{q^{\prime}} \alpha_{s}^{\prime}} \frac{1}{8 \pi}\left(m_{q^{\prime}} \alpha_{s}^{\prime}\right)^{3} .
$$

The scattering cross section between a mirror quarkonium $\chi_{i}^{\prime}$ and $f$ is given by

$$
\sigma v_{\mathrm{rel}}\left(f \chi_{i}^{\prime} \rightarrow f \chi_{i}^{\prime}\right)=\frac{1}{8 \pi}\left(\frac{4}{11}\right)^{2}\left(\frac{m_{f}}{v}\right)^{2}\left(\frac{v m_{\chi_{i}^{\prime}}}{v^{\prime 2}}\right)^{2} \frac{m_{f} m_{\chi_{i}^{\prime}}}{m_{f}+m_{\chi_{i}^{\prime}}} \frac{p_{\mathrm{cm}}}{m_{h}^{4}} .
$$

Here we assume that the mass of the quarkonium is dominated by the mirror quark mass. We take into account the scattering of all the quarkonia composed of $d^{\prime}, s^{\prime}, b^{\prime}, u^{\prime}, c^{\prime}$ with spin-CP $0^{+-}(\eta$-like $)$ and $1^{--}(J / \psi$-like $)$.

In figure 11 we show by solid lines the decoupling temperature $T_{d, \text { had }}$ determined by the Higgs exchange in the hadron picture. In some of the parameter space $T_{d \text {,had }}$ is lower than $T_{c}^{\prime}$. The decoupling temperature is dominantly determined by the decay of glueballs. The estimated $T_{d, \text { had }}$ is however close to $T_{c}^{\prime}$, and the thermal effect may be important (e.g. that on the glueball mass). The raise of $T_{d, \text { had }}$ when lowering $\epsilon^{\prime} / \epsilon$ below about 2.6 is due to the kinematic suppression of the decay of the lightest glueball into $b \bar{b}$. Inclusion of higher resonances might make $T_{d, h a d}$ smaller than $T_{c}^{\prime}$ also for $\epsilon^{\prime} / \epsilon \lesssim 2.6$. 


\subsubsection{Decoupling temperature from kinetic mixing}

The kinetic mixing between the hypercharge gauge fields,

$$
\frac{1}{2} \frac{\epsilon_{\mathrm{kin}}}{\cos \theta_{W}^{2}} B^{\mu \nu} B_{\mu \nu}^{\prime},
$$

can maintain thermal equilibrium between the SM and mirror sectors through the scattering between a mirror charged fermion and the SM photon. The mirror electron is the lightest mirror charged fermion and decoupling does not occur until the temperature drops below its mass. For $T \ll m_{e^{\prime}}$, the scattering cross section for the process $e^{\prime} \gamma^{\prime} \leftrightarrow e^{\prime} \gamma$ is given by

$$
\sigma\left(f^{\prime} \gamma^{\prime} \leftrightarrow f^{\prime} \gamma\right) v=\frac{8 \pi}{3} \epsilon_{\mathrm{kin}}^{2} \alpha^{2} \frac{1}{m_{e^{\prime}}^{2}}
$$

The scattering rate becomes smaller than the expansion rate of the universe below a temperature $T_{d, k i n}$,

$$
T_{d, k i n} \simeq \frac{m_{e^{\prime}}}{4+2 \ln \frac{\epsilon_{\text {kin }}}{10^{-6}}} .
$$

Sufficient suppression of $\Delta N_{\text {eff }}$ requires $0.2 \mathrm{GeV}<T_{d, k i n}<T_{c}^{\prime}$. For the mirror electron mass we are interested in, this is achieved for $\epsilon_{\text {kin }} \sim 10^{-7}-10^{-6}$. Kinetic mixing of this size is excluded if dark matter is mirror atoms, but is allowed if dark matter is composed of mirror neutrons [5].

\section{Variant models}

While in principle there are many models based on eqs. (2.2), (2.3), they are greatly restricted by the need to account for the known fermion masses and quark mixings. To illustrate the broad persistence, given this constraint, of the mirror fermion spectrum obtained in section IIIA, we briefly consider in this section two variants of the SU(5)compatible model examined there. In both the new models [66] we take the FN charge of the $\mathcal{Q}_{1}$ multiplet to deviate by one unit from the charge of $\bar{u}_{1}, \bar{e}_{1}$ in order to get the same scaling law in terms of $\epsilon$ as in the Volfenstein parameterization of the CKM angles, $V_{u s} \approx \lambda_{c}, V_{c b} \approx \lambda_{c}^{2}, V_{u b} \approx \lambda_{c}^{3}$, in terms of $\lambda_{c}=0.22$.

The FN charges of the two models and the corresponding scaling law of the masses are:

- Model B1

$$
Q:(3,2,0), \bar{u}:(4,2,0), \bar{e}:(4,2,0), \bar{d}, L:(4,3,3)
$$

$$
\begin{aligned}
& m_{t} \sim 1+O\left(\epsilon^{4}\right), \quad \quad m_{c} \sim \epsilon^{4}\left(1+O\left(\epsilon^{4}\right)\right), \quad m_{u} \sim \epsilon^{7}\left(1+O\left(\epsilon^{4}\right)\right) \\
& m_{b} \sim \epsilon^{3}\left(1+O\left(\epsilon^{2}\right)\right), \quad m_{s} \sim \epsilon^{5}\left(1+O\left(\epsilon^{2}\right)\right), \quad m_{d} \sim \epsilon^{7}\left(1+O\left(\epsilon^{2}\right)\right) \\
& m_{\tau} \sim \epsilon^{3}\left(1+O\left(\epsilon^{2}\right)\right), \quad m_{\mu} \sim \epsilon^{5}\left(1+O\left(\epsilon^{2}\right)\right), \quad m_{e} \sim \epsilon^{8}\left(1+O\left(\epsilon^{2}\right)\right)
\end{aligned}
$$



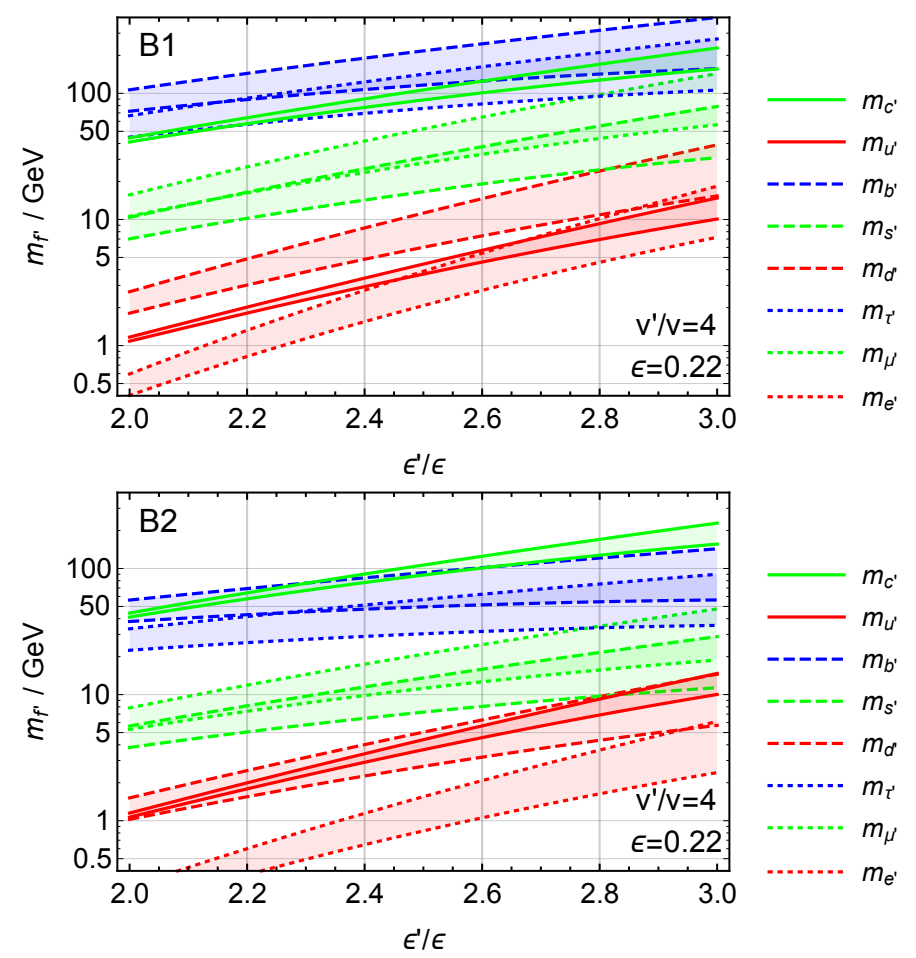

Figure 12. The mass spectrum of the mirror fermions in models B1, B2. The bands show a possible deviation from the simple scaling law with $|\delta|<1$. Here we have taken the central value for the Yukawa couplings of the SM fermions.

- Model B2

$$
\begin{array}{lll}
Q:(3,2,0), & \bar{u}:(4,2,0), \bar{e}:(4,2,0), \bar{d}, L:(3,2,2) \\
m_{t} \sim 1+O\left(\epsilon^{4}\right), & m_{c} \sim \epsilon^{4}\left(1+O\left(\epsilon^{4}\right)\right), & m_{u} \sim \epsilon^{7}\left(1+O\left(\epsilon^{4}\right)\right) \\
m_{b} \sim \epsilon^{2}\left(1+O\left(\epsilon^{2}\right)\right), & m_{s} \sim \epsilon^{4}\left(1+O\left(\epsilon^{2}\right)\right), & m_{d} \sim \epsilon^{6}\left(1+O\left(\epsilon^{2}\right)\right) \\
m_{\tau} \sim \epsilon^{2}\left(1+O\left(\epsilon^{2}\right)\right), & m_{\mu} \sim \epsilon^{4}\left(1+O\left(\epsilon^{2}\right)\right), & m_{e} \sim \epsilon^{7}\left(1+O\left(\epsilon^{2}\right)\right)
\end{array}
$$

How well these models account for the known masses and mixings is illustrated in appendix C, where they are also compared with the SU(5)-compatible model of section IIIA.

Based on eq. (3.3), similarly to figure 1, we show in figure 12 the masses of the mirror fermions. The consistency of these models with the constraints from Higgs decays is shown in figure 13. Concerning Dark Matter, the overlap of the masses of $u^{\prime}, d^{\prime}$ in figure 12 for the model B2 makes it relatively more likely that in this case $B_{u d d}^{\prime}$ be the lightest stable mirror baryon.

\section{Conclusions}

Can Minimal Mirror Twin Higgs be the reason why LHC has not found, so far, any signal of New Physics and, at the same time, explain the surprising similar size of Dark Matter and 

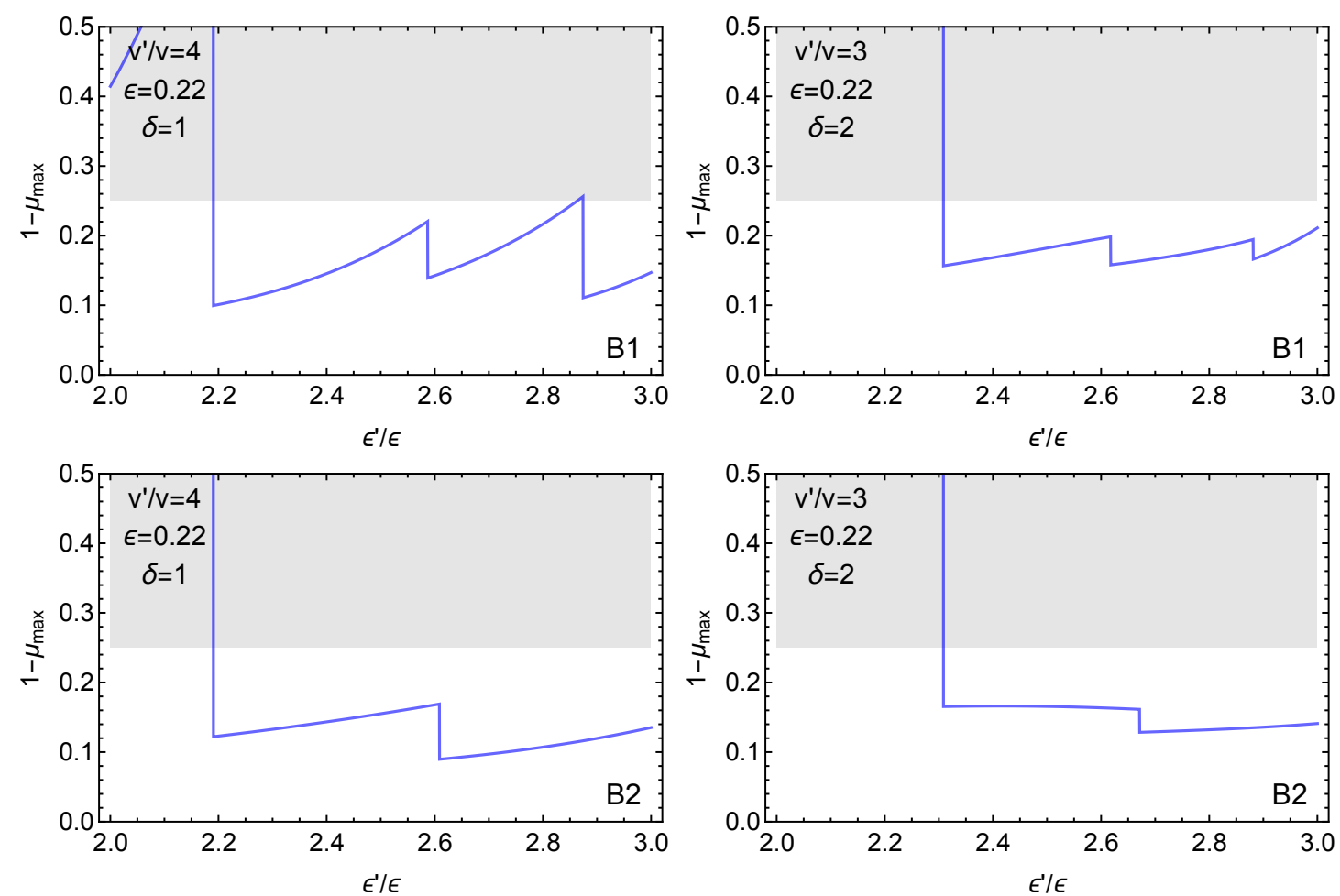

Figure 13. Prediction of the Higgs signal strength in models B1, B2. Panels with $\delta=1,2$ have the mass spectrum of mirror fermions chosen to minimize the invisible decay of the Higgs. Decays to $c^{\prime}$ exclude $\epsilon^{\prime} / \epsilon$ less than about 2.2.

baryon densities? In [5] we have argued in favour of this possibility, attributing the needed breaking of parity only to a difference in the Yukawa couplings between standard and mirror fermions, except the top. We were led to this hypothesis by the need to keep under control, in the absence of an exotic cosmological history, the amount of mirror radiation.

In this paper we have made the further step of identifying the source of the difference in the standard and mirror Yukawa couplings: a different single scaling parameter, $\epsilon$ versus $\epsilon^{\prime}$, that is at the origin of the hierarchy in the masses of the charged fermions. In this way the masses of the light mirror fermions are raised, while the top Yukawa couplings remain similar, and the separation between the heaviest and the lightest is reduced, with respect to the masses of the standard fermions, by almost two orders of magnitude. This can be done in a general scheme that we call "Minimal Flavor Hierarchy". While there can be many such models, different in the physical origin and in the detailed parameters, the range of the predicted signals is greatly reduced by the need to reproduce the known charged fermion masses. Therefore, although we have based our detailed predictions on a specific Froggatt-Nielsen model with SU(5)-compatible U(1) charges, we believe that their main features have a broader validity.

From a phenomenological point of view the new main achievement in the present paper is contained in the part of section 3 where we discuss the various DM configurations, which can be in the form of mirror atoms, Hydrogen-like or Helium-like, or of mirror neutrons. A 
special summary of the overall situation is in figure 9. It is remarkable that one can give a detailed prediction of the possible DM configurations and that the entire allowed regions, mostly controlled by the single parameter $\epsilon^{\prime} / \epsilon$, are within reach of foreseen direct detection experiments for a wide range of the uncertainties. As already pointed out in [5] we expect other correlated signals in Higgs decays and in the amount of dark radiation. In theories with Minimal Flavor Hierarchies these predictions are sharpened, as shown in figures 2, 13 and figure 11 respectively.

\section{Acknowledgments}

The work of L.H. and K.H. was supported in part by the Director, Office of Science, Office of High Energy and Nuclear Physics, of the US Department of Energy under Contract DE-AC02-05CH11231 and by the National Science Foundation under grants PHY-1316783 and PHY-1521446.

\section{A Minimal flavor hierarchy from extra dimensions}

We first review the model of the flavor hierarchy introduced in [10]. We consider a flat extra dimension compactified to an orbifold $S_{1} / Z_{2}$, with fixed points $y=0, \pm L / 2$. For a fermion $\psi$ the following boundary condition is imposed to obtain a chiral fermion in the low energy $4 \mathrm{D}$ theory,

$$
\psi(x,-y)=i \gamma_{5} \psi(x, y), \quad \psi\left(x, \frac{L}{2}+y\right)=i \gamma_{5} \psi\left(x, \frac{L}{2}-y\right), \quad \gamma_{5}=-i\left(\begin{array}{cc}
1 & \\
& -1
\end{array}\right) .
$$

The fermion $\psi$ has a mass term with a non-trivial profile in the extra dimension,

$$
\mathcal{L}_{5 D}=\bar{\psi}\left(i \gamma^{N} \partial_{N}-m(y)\right) \psi, m(y)=\left\{\begin{array}{l}
M \quad: 0<y<L / 2 \\
-M:-L / 2<y<0 .
\end{array}\right.
$$

The profile is consistent with the boundary condition as well as with the $Z_{2}$ symmetry, and may be dynamically generated with a thin domain wall of a scalar field. The equation of motion of the wave function of the zero-mode of $\psi$ is given by

$$
\partial_{y} \psi_{0, \pm}= \pm M \psi_{0, \pm}
$$

The solution for this equation is symmetric for $y \leftrightarrow-y$ due to the profile of $m(y)$, and only $\psi_{+, 0}$ is consistent with the boundary condition. The normalized zero mode wave function is given by

$$
\psi_{0}(y)=\sqrt{\frac{2 M}{e^{M L}-1}} e^{M y} .
$$

The zero mode is localized around $y=0$ for $M<0$, and around $y=L / 2$ for $M>0$. 


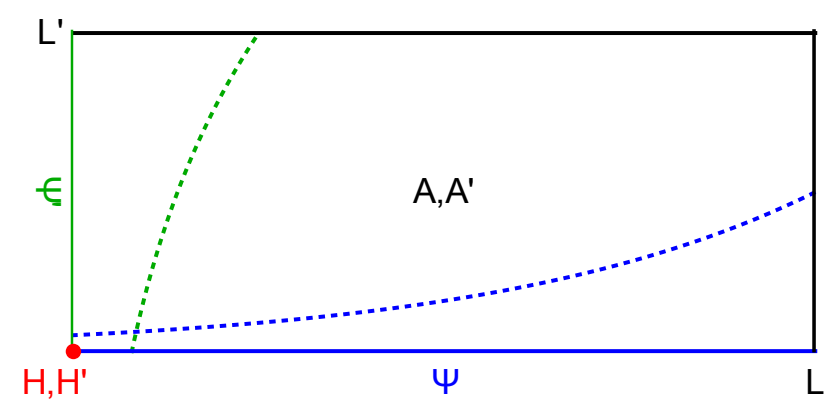

Figure 14. Sketch of a $6 \mathrm{D}$ theory that leads to the MMTH scenario.

The structure of the Yukawa couplings in eq. (2.2) arises when the SM fermions, with bulk mass $M_{i}$ different from each other, are localized around $y=L / 2$, while the Higgs field is confined to the brane at $y=0$. From the $5 \mathrm{D}$ brane couplings

$$
\mathcal{L}_{5 D}=-\delta(y) \frac{\lambda_{i j}}{M_{*}} H f_{L, i} \bar{f}_{R, j},
$$

we obtain the $4 \mathrm{D}$ Yukawa couplings

$$
\mathcal{L}_{4 D}=-y_{i j} H f_{L, i} \bar{f}_{R, j}, \quad y_{i j}=\frac{\lambda_{i j}}{M_{*}} \psi_{f_{L, i i}, 0}(0) \psi_{\bar{f}_{R, j}, 0}(0) \propto e^{-M_{i} / L} \lambda_{i j} e^{-\bar{M}_{j} / L}
$$

The $O(1)$ top yukawa coupling is obtained by localizing $Q_{3}$ and $\bar{u}_{3}$ at $y=0$.

To obtain the minimal flavour hierarchy of MMTH, as described in section 2, we consider the $6 \mathrm{D}$ configuration depicted in figure 14. The extra dimensions are compactified to $T /\left(Z_{2} \times Z_{2}\right)$, with fixed points at $\left(y_{5}, y_{6}\right)=(0,0),(L / 2,0),\left(0, L^{\prime} / 2\right)$ and $\left(L / 2, L^{\prime} / 2\right)$. The SM and mirror fermions are confined to the $5 \mathrm{D}$ brane $y_{6}=0$ and $y_{5}=0$ respectively. Those fermions have exponential profiles in each $5 \mathrm{D}$ brane via the mechanism shown above. The Higgs sector is confined to the $4 \mathrm{D}$ brane at $\left(y_{5}, y_{6}\right)=(0,0)$. The $Z_{2}$ symmetry, which is now understood as the symmetry $y_{5} \leftrightarrow y_{6}$, is spontaneously broken by $L^{\prime}<L$, which gives $\epsilon^{\prime}>\epsilon$.

We assume that the gauge fields live in the $6 \mathrm{D}$ bulk which ensures the identity of the gauge couplings from the $6 \mathrm{D}$ bulk, $g=g^{\prime}$, at the tree level. A difference between them could arise from the quantum correction from KK modes and the 5D bulk gauge couplings. The former is loop suppressed and is much smaller than the tree level one unless the cut off scale is much larger than the KK scale. The latter is also suppressed if $L, L^{\prime} \gtrsim M_{*}^{-1}$, where $M_{*}$ is the cut off scale, due to the volume factor. It is also possible to obtain nonMinimal Mirror Twin Higgs with $g \neq g^{\prime}$ with the above two corrections, or confining gauge fields to the $5 \mathrm{D}$ bulks. This might be beneficial for two reasons. First, $g_{3}^{\prime}>g_{3}$ raises the mirror QCD phase transition temperature $T_{c}^{\prime}$, which helps suppressing the abundance of dark radiation. Second, $\alpha^{\prime}>\alpha$ makes recombination for the mirror atomic dark matter more efficient. It also suppresses the self-interaction of atomic dark matter, widening the allowed parameter range. We do not pursue this possibility in the present paper. 
So far we have treated the Higgs field as a fundamental field. In some UV completions of MMTH the Higgs could be composite. Then the above derivation of the suppression factor $e^{-M L}$ should be applied to the operators which eventually lead to the SM Yukawa couplings. For example, if the Yukawa couplings originate from mixing between fundamental SM fermions and composite fermions, we may apply the above discussion to the mixing instead of the Yukawa couplings in eq. (A.5).

\section{B Scaling law}

The down Yukawa matrix for the SU(5) compatible model in eq. (3.1) is of the form

$$
\mathcal{L}=H^{*} Q_{i} Y_{d, i j} \bar{d}_{j}, \quad Y_{d, i j}=\epsilon^{3}\left(\begin{array}{ccc}
a \epsilon^{5} & b \epsilon^{4} & c \epsilon^{4} \\
d \epsilon^{3} & e \epsilon^{2} & f \epsilon^{2} \\
g \epsilon^{1} & h & i
\end{array}\right)
$$

The square of the matrix is

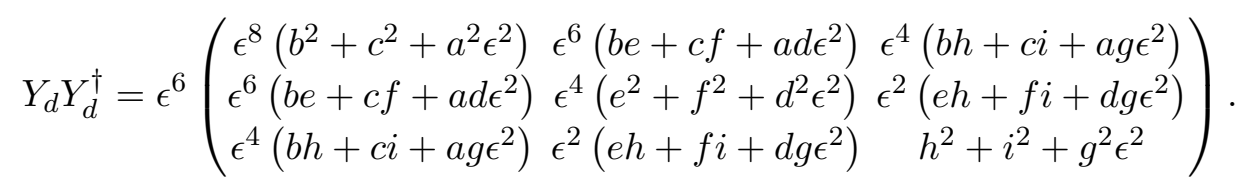

From this we obtain the bottom Yukawa coupling,

$$
y_{b}^{2}=\left(h^{2}+i^{2}\right) \epsilon^{6}\left(1+O\left(\epsilon^{2}\right)\right) .
$$

By integrating out the bottom quark, the $2 \times 2$ squared Yukawa matrix of the first two generations is given by

$$
\begin{aligned}
\left(Y_{d} Y_{d}^{\dagger}\right)_{d s, 11} \simeq & \frac{(c h-b i)^{2}}{h^{2}+i^{2}} \epsilon^{14}\left(1+\frac{\epsilon^{2}\left(-a\left(h^{2}+i^{2}\right)+b g h+c g i\right)^{2}}{\left(h^{2}+i^{2}\right)(c h-b i)^{2}}\right), \\
\left(Y_{d} Y_{d}^{\dagger}\right)_{d s, 12} \simeq & \frac{(c h-b i)(f h-e i)}{h^{2}+i^{2}} \epsilon^{12} \\
& \times\left(1+\frac{\left(-a\left(h^{2}+i^{2}\right)+b g h+c g i\right)\left(-d\left(h^{2}+i^{2}\right)+e g h+f g i\right)}{\left(h^{2}+i^{2}\right)(c h-b i)(f h-e i)} \epsilon^{2}\right), \\
\left(Y_{d} Y_{d}^{\dagger}\right)_{d s, 22} \simeq & \frac{(f h-e i)^{2}}{h^{2}+i^{2}} \epsilon^{10}\left(1+\frac{\left(-d\left(h^{2}+i^{2}\right)+e g h+f g i\right)^{2}}{\left(h^{2}+i^{2}\right)(f h-e i)^{2}} \epsilon^{2}\right) .
\end{aligned}
$$

Therefore the Yukawa coupling of the strange quark is

$$
y_{s}^{2}=\frac{(f h-e i)^{2}}{h^{2}+i^{2}} \epsilon^{10}\left(1+O\left(\epsilon^{2}\right)\right)
$$

and, by integrating $s$ out, we obtain

$$
y_{d}^{2}=\frac{(-a e i+a f h+b d i-b f g-c d h+c e g)^{2}}{(f h-e i)^{2}} \epsilon^{16}\left(1+O\left(\epsilon^{2}\right)\right) .
$$




\begin{tabular}{|l|c|c|c|c|c|c|c|c|}
\hline model & $\frac{m_{b}}{m_{t}}$ & $\frac{m_{\tau}}{m_{t}}$ & $\frac{m_{c}}{m_{t}}$ & $\frac{m_{s}}{m_{t}}$ & $\frac{m_{\mu}}{m_{t}}$ & $\frac{m_{u}}{m_{t}}$ & $\frac{m_{d}}{m_{t}}$ & $\frac{m_{e}}{m_{t}}$ \\
\hline $\mathrm{SU}(5)$ & $1.6 \epsilon^{3}$ & $1.1 \epsilon^{3}$ & $1.8 \epsilon^{4}$ & $1.0 \epsilon^{5}$ & $1.25 \epsilon^{5}$ & $2.5 \epsilon^{8}$ & $4.5 \epsilon^{8}$ & $0.6 \epsilon^{8}$ \\
\hline $\mathrm{B} 1$ & $1.6 \epsilon^{3}$ & $1.1 \epsilon^{3}$ & $1.8 \epsilon^{4}$ & $1.0 \epsilon^{5}$ & $1.25 \epsilon^{5}$ & $0.55 \epsilon^{7}$ & $1.0 \epsilon^{7}$ & $0.6 \epsilon^{8}$ \\
\hline $\mathrm{B} 2$ & $0.5 \epsilon^{2}$ & $0.4 \epsilon^{2}$ & $4.0 \epsilon^{4}$ & $0.45 \epsilon^{4}$ & $0.6 \epsilon^{4}$ & $2.2 \epsilon^{7}$ & $0.7 \epsilon^{6}$ & $0.5 \epsilon^{7}$ \\
\hline
\end{tabular}

Table 3. Leading scaling terms for the charged fermion masses in: i) $\mathrm{SU}(5)$, with $\epsilon=0.22$; ii) model B1, with $\epsilon=0.22$; iii) model B2, with $\epsilon=0.18$.

\begin{tabular}{|l|c|c|c|}
\hline model & $V_{u s}$ & $V_{c b}$ & $V_{u b}$ \\
\hline $\mathrm{SU}(5)$ & $4.5 \epsilon^{2}$ & $1.0 \epsilon^{2}$ & $2.3 \epsilon^{4}$ \\
\hline $\mathrm{B} 1$ & $1.0 \epsilon$ & $1.0 \epsilon^{2}$ & $0.5 \epsilon^{3}$ \\
\hline $\mathrm{B} 2$ & $1.2 \epsilon$ & $1.5 \epsilon^{2}$ & $1.8 \epsilon^{3}$ \\
\hline
\end{tabular}

Table 4. Leading scaling terms for the CKM mixings in: i) $\mathrm{SU}(5)$, with $\epsilon=0.22$; ii) model B1, with $\epsilon=0.22$; iii) model B2, with $\epsilon=0.18$.

\section{Evidence for the minimal flavor hierarchy}

How well does the flavor structure of (2.2) account for the observed hierarchies of quark and charged lepton masses in the three FN models considered in this paper? With $\epsilon$ of about 0.2 and relative corrections of order $\epsilon^{2}$ or smaller, the leading scaling terms in eq. (3.2) give a quite accurate approximation for the charged fermion masses and quark mixing angles in the SM. These leading terms are shown in table 3 and 4 for the models considered in the text by fitting the experimental numbers without subleading corrections. In the $\mathrm{SU}(5)$ model the coefficients of the leading terms shown in the tables are determined by a single scaling variable, taken to be $\epsilon=0.22$, and five integers. In model B1 we take $\epsilon=0.22$ and in model B2 $\epsilon=0.18$.

The closeness to unity of the coefficients of the leading scaling terms shown in table 3 and 4 represents evidence for the FN picture of the flavour parameters. The neutrino masses and the PMNS angles can also be described by extending the models discussed in the text with right handed neutrinos $[27,66]$.

\section{Mirror matter asymmetry for $\boldsymbol{m}_{u^{\prime}} \sim \boldsymbol{m}_{\boldsymbol{d}^{\prime}}$}

As commented in section IIIC6, it is natural to assume that the mirror sector has nonzero baryon and lepton asymmetries similar to the SM ones. As the universe cools, the symmetric components annihilate and almost disappear, and only the asymmetric components remain. The dark matter component is determined by the scattering of the following particles,

$$
B_{u u u}^{\prime}, B_{u u d}^{\prime}, B_{u d d}^{\prime}, B_{d d d}^{\prime}, e^{\prime}, \nu^{\prime}
$$


Let us first consider the $B_{u u d}^{\prime} \equiv p^{\prime}, B_{u d d}^{\prime} \equiv n^{\prime}, e^{\prime}$ and $\nu^{\prime}$ system. For simplicity we drop the superscript ' from now on. The corresponding number densities are given by

$$
\begin{array}{rlrl}
n_{p} & =2\left(\frac{m_{p} T}{2 \pi}\right)^{3 / 2} e^{-m_{p} / T+\mu_{p} / T}, & & n_{\bar{p}}=2\left(\frac{m_{p} T}{2 \pi}\right)^{3 / 2} e^{-m_{p} / T-\mu_{p} / T}, \\
n_{n} & =2\left(\frac{m_{n} T}{2 \pi}\right)^{3 / 2} e^{-m_{n} / T+\mu_{n} / T}, & n_{\bar{n}} & =2\left(\frac{m_{n} T}{2 \pi}\right)^{3 / 2} e^{-m_{n} / T-\mu_{n} / T}, \\
n_{e} & =2\left(\frac{m_{e} T}{2 \pi}\right)^{3 / 2} e^{-m_{e} / T+\mu_{e} / T}, & n_{\bar{e}} & =2\left(\frac{m_{e} T}{2 \pi}\right)^{3 / 2} e^{-m_{e} / T-\mu_{e} / T}, \\
n_{\nu} & \simeq \frac{3 \zeta(3)}{4 \pi^{2}} T^{3}+\frac{1}{12} T^{3} \frac{\mu_{\nu}}{T}, & n_{\bar{\nu}} \simeq \frac{3 \zeta(3)}{4 \pi^{2}} T^{3}-\frac{1}{12} T^{3} \frac{\mu_{\nu}}{T} .
\end{array}
$$

The asymmetries are given by

$$
\begin{aligned}
\Delta_{p} & \equiv \frac{n_{p}-n_{\bar{p}}}{T^{3}}=4\left(\frac{m_{p}}{2 \pi T}\right)^{3 / 2} e^{-m_{p} / T} \sinh \frac{\mu_{p}}{T} \\
\Delta_{n} & \equiv \frac{n_{n}-n_{\bar{n}}}{T^{3}}=4\left(\frac{m_{n}}{2 \pi T}\right)^{3 / 2} e^{-m_{n} / T} \sinh \frac{\mu_{n}}{T} \\
\Delta_{e} & \equiv \frac{n_{e}-n_{\bar{e}}}{T^{3}}=4\left(\frac{m_{e}}{2 \pi T}\right)^{3 / 2} e^{-m_{e} / T} \sinh \frac{\mu_{e}}{T} \\
\Delta_{\nu} & \equiv \frac{n_{\nu}-n_{\bar{\nu}}}{T^{3}}=\frac{1}{6} \frac{\mu_{\nu}}{T}
\end{aligned}
$$

The charge neutrality condition, the conservation of the baryon asymmetry $B \equiv\left(n_{B}-\right.$ $\left.n_{\bar{B}}\right) / T^{3}$, and that of the lepton asymmetry $L \equiv\left(n_{L}-n_{\bar{L}}\right) / T^{3}$ require that

$$
\begin{aligned}
\Delta_{e} & =\Delta_{p}, \\
\Delta_{n} & =B-\Delta_{p}, \\
\mu_{\nu} / T & =6 L-6 \Delta_{p} .
\end{aligned}
$$

The charged current interactions maintain

$$
\mu_{p}+\mu_{e}=\mu_{n}+\mu_{\nu}
$$

up to some decoupling temperature $T_{d, W}$. The reaction $p+e \rightarrow n+\nu$ changes the asymmetry of $p$ and $e$ with a rate

$$
\begin{aligned}
\frac{d}{d t} \Delta_{p} & =-\sigma v(p+e \rightarrow n+\nu) \frac{n_{p} n_{e}-n_{\bar{p}} n_{\bar{e}}}{\Delta_{p}}, \\
\sigma v(p+e \rightarrow n+\nu) & =\frac{1}{8 \pi} \frac{\left(m_{p}+m_{e}-m_{n}\right)^{2}}{v^{4}}
\end{aligned}
$$

For $T \lesssim m_{p} / 25, n_{\bar{p}}$ is smaller than $n_{p}$, and we obtain

$$
\frac{\frac{d}{d t} \Delta_{p}}{\Delta_{p}} \simeq \sigma v(p+e \rightarrow n+\nu) n_{e} \simeq-\sigma v(p+e \rightarrow n+\nu) 2\left(\frac{m_{e}}{2 \pi T}\right)^{3 / 2} e^{-m_{e} / T}
$$


Here we assume that the symmetric component of $e$ dominates over the asymmetric one. The decoupling temperature of the process is given by $\left(d \Delta_{p} / d t\right) / \Delta_{p}(T)=H(T)$. We find

$$
T_{d, W} \simeq \frac{m_{e}}{18}
$$

At this temperature the asymmetric component of the mirror electrons is smaller than the symmetric one, as assumed. Furthermore, since $m_{p}>m_{e}$, it is indeed verified that $n_{\bar{p}}$ is much smaller than $n_{p}$.

The dominance of the symmetric component of $e$ implies $\left|\mu_{e} / T\right| \ll 1$. Eq. (D.12) shows that $\mu_{\nu} / T \simeq 6 L$. On the other hand, at least one of $\mu_{n} / T$ and $\mu_{p} / T$ must be much larger than unity to maintain the baryon asymmetry. Thus eq. (D.13) is solved by $\mu_{p}=\mu_{n}+6 L T$. We therefore obtain the relative abundance of $p$ and $n$,

$$
\frac{\Delta_{n}}{\Delta_{p}}=e^{\left(m_{p}-m_{n}\right) / T} e^{-6 L}\left(\frac{m_{n}}{m_{p}}\right)^{3 / 2} .
$$

Except for that case with $m_{p} \simeq m_{n},\left|m_{p}-m_{n}\right| / T_{d, W}$ is much larger than unity. For $|L| \ll 1$, the baryon asymmetry is stored in the lighter between $p$ and $n$. If $L=O(1)$ this conclusion may be changed, but we do not pursue this possibility in this paper.

One can repeat the same analysis including all baryons $B_{u u u}^{\prime}, B_{u u d}^{\prime}, B_{u d d}^{\prime}, B_{d d d}^{\prime}$, and show that the chemical potentials of those four baryons are the same. We conclude that the mirror baryon asymmetry is stored in the lightest among $B_{u u u}^{\prime}, B_{u u d}^{\prime}, B_{u d d}^{\prime}$ and $B_{d d d}^{\prime}$, as anticipated in section IIIC1.

\section{E Mirror recombination with electron capture}

In the following we drop the superscript ' for simplicity. We consider the situation where $m_{p}+m_{e}>m_{n}$, so that the mirror atom is unstable due to the mirror electron capture process, $p+e \rightarrow n+\nu$. For s-orbit states, the decay rate of a mirror atom is given by

$$
\begin{aligned}
\Gamma(H(n s) \rightarrow n+\nu) & =|\psi(0)|^{2} \sigma v(p+e \rightarrow n+\nu)=\frac{\left(m_{e} \alpha\right)^{3}}{n^{5} \pi} \frac{1}{8 \pi} \frac{\left(m_{p}+m_{e}-m_{n}\right)^{2}}{v^{4}} \\
& \simeq 2 \times 10^{-20} \mathrm{GeV}\left(\frac{4}{v^{\prime} / v}\right)^{4}\left(\frac{m_{e}}{1 \mathrm{GeV}}\right)^{3}\left(\frac{m_{p}+m_{e}-m_{n}}{1 \mathrm{GeV}}\right)^{2} \frac{1}{n^{5}}
\end{aligned}
$$

Around the temperature where mirror recombination occurs, $T \lesssim m_{e} \alpha^{2}$, the decay rate of the mirror atom is much larger than the Hubble expansion rate, and electron capture is expected to affect the recombination process.

We formulate recombination with electron capture by modifying the Peebles model [67]. We consider transitions between the $1 s, 2 s$ and $2 p$ atomic states as well as the ionized states. The differential equation governing their fractions, $x_{1} \equiv n_{1 s} / n_{\mathrm{DM}}, x_{2} \equiv\left(n_{2 s}+n_{2 p}\right) / n_{\mathrm{DM}}$, 
$x_{e} \equiv n_{e} / n_{\mathrm{DM}}$, are given by

$$
\begin{aligned}
\dot{x_{e}}= & -\left(x_{e}^{2} n_{\mathrm{DM}} \alpha_{1}-x_{1} \beta_{1}\right) P_{1 s}-\left(x_{e}^{2} n_{\mathrm{DM}} \alpha_{B}-x_{2} \beta_{B}\right) \\
\dot{x_{1}}= & +\left(x_{e}^{2} n_{\mathrm{DM}} \alpha_{1}-x_{1} \beta_{1}\right) P_{1 s}+\frac{3}{4} \Gamma_{2 p 1 s} P_{2 s 1 s}\left(x_{2}-4 x_{1} e^{-E_{2 s 1 s} / T}\right) \\
& +\frac{1}{4} \Gamma_{2 s 1 s}\left(x_{2}-4 x_{1} e^{-E_{2 s 1 s} / T}\right)-x_{1} \Gamma_{1 s, e c} \\
\dot{x_{2}}= & +\left(x_{e}^{2} n_{\mathrm{DM}} \alpha_{B}-x_{2} \beta_{B}\right)-\frac{3}{4} \Gamma_{2 p 1 s} P_{2 s 1 s}\left(x_{2}-4 x_{1} e^{-E_{2 s 1 s} / T}\right) \\
& -\frac{1}{4} \Gamma_{2 s 1 s}\left(x_{2}-4 x_{1} e^{-E_{2 s 1 s} / T}\right)-\frac{1}{4} x_{2} \Gamma_{2 s, e c}
\end{aligned}
$$

and satisfies the detailed balance relation if electron capture is absent.

Recombination into the ground state. The first terms in the r.h.s. of eqs. (E.2) and (E.3) are from the process $p+e \leftrightarrow H(1 s)+\gamma$. The coefficient $\alpha_{1}$ is the thermal average of the cross section times the velocity of the process $p+e \rightarrow H(1 s)+\gamma$, which we extract from [68] by subtracting the case $\mathrm{B}$ coefficient from the case $\mathrm{A}$ one. The coefficient $\beta_{1}$ is given by

$$
\beta_{1}=\left(\frac{m_{e} T}{2 \pi}\right)^{3 / 2} e^{-E_{1 s} / T} \alpha_{1},
$$

where $E_{1 s}$ is the binding energy of the 1 s state. $P_{1 s}$ is the probability that the emitted photon escapes from the capture by the inverse process and is given by the optical depth $\tau_{1 s}$ as

$$
P_{1 s}=\frac{1-e^{-\tau_{1 s}}}{\tau_{1 s}}, \quad \tau_{1 s}=\frac{x_{1} n_{\mathrm{DM}}}{H} \frac{\pi^{2} \alpha_{1}}{E_{1 s}^{3}}\left(\frac{m_{e} T}{2 \pi}\right)^{3 / 2} .
$$

When electron capture is absent, as recombination proceeds the optical width is so large that the process $p+e \leftrightarrow H(1 s)+\gamma$ does not contribute to recombination. With electron capture, $x_{1}$ remains very small and the optical depth is almost zero, and we may use the approximation $P_{1 s} \simeq 1$.

Recombination into excited states. The second term in the r.h.s. of eq. (E.2) is the effect of the process $p+e \leftrightarrow H(n>1)+\gamma$. The $n>2$ states rapidly cascade down to the $n=2$ states, and we may use the following so-called case-B coefficient for the evolution of $x_{2}$,

$$
\alpha_{B} \equiv 1.14 \times \sum_{n=2}^{\infty} \sum_{l=0}^{n-1} \sum_{m=-l}^{l}\langle\sigma v(p+e \rightarrow H(n l m)+\gamma)\rangle_{\text {thermal }} .
$$

The factor of 1.14 allows the Peebles approximation to agree with a multi-level calculation [48]. The coefficient $\beta_{B}$ is given by

$$
\beta_{B}=\left(\frac{m_{e} T}{2 \pi}\right)^{3 / 2} e^{-E_{1 s} / T} \alpha_{B}
$$




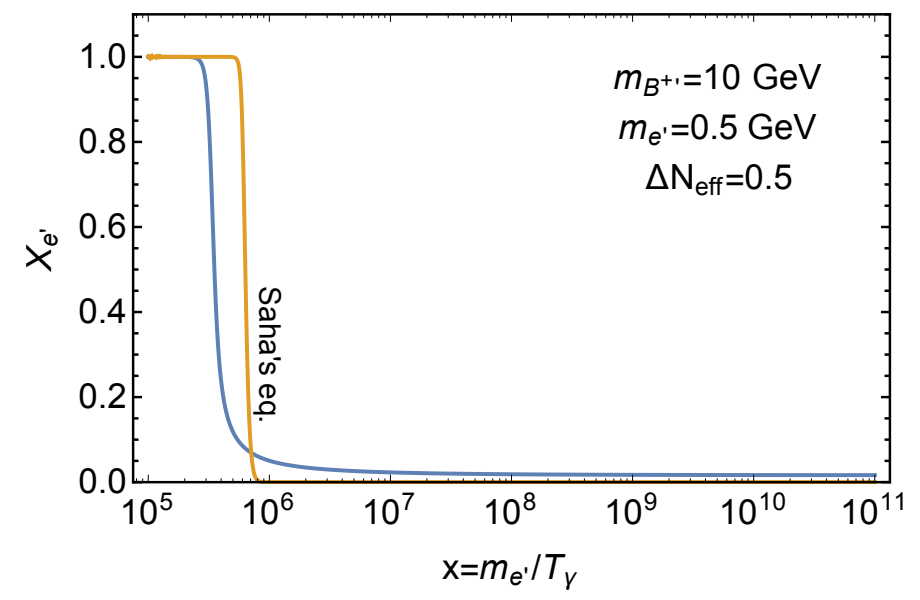

Figure 15. A sample evolution of the ionization fraction when electron capture occurs.

Lyman- $\boldsymbol{\alpha}$ decay $\mathbf{2 p} \rightarrow \mathbf{1 s}$. The second terms in the r.h.s. of eqs. (E.3) and (E.4) are the effect of the process $H(2 p) \leftrightarrow H(1 s)+\gamma$, and $\Gamma_{2 p 1 s}$ is the decay width of this process. $E_{2 s 1 s}$ is the difference of the energy levels of the $n=2$ and 1 states. $P_{2 p 1 s}$ is the probability that the emitted photon escapes from the capture by the inverse process, and is given by the optical depth $\tau_{2 p 1 s}$ as

$$
P_{2 p 1 s}=\frac{1-e^{-\tau_{2 p 1 s}}}{\tau_{2 p 1 s}}, \quad \tau_{2 p 1 s}=\frac{x_{1} n_{\mathrm{DM}}}{H} \frac{3 \pi^{2} \Gamma_{2 p 1 s}}{E_{1 s}^{3}} .
$$

As is the case with recombination to the ground state, we may use the approximation $P_{2 p 1 s} \simeq 1$.

Two-photon decay. The third terms in the r.h.s. of eqs. (E.3) and (E.4) are the effect of the process $H(2 s) \leftrightarrow H(1 s)+2 \gamma$, and $\Gamma_{2 s 1 s}$ is the decay width of this process. Without electron capture, the two-photon decay may dominate over the Lyman- $\alpha$ decay, due to the large optical depth $\tau_{2 p 1 s}$. With electron capture, the two-photon decay is negligible, and we ignore it.

Electron capture. The last terms in the r.h.s. of eqs. (E.3) and (E.4) are the effect of the process $H(2 s, 1 s) \rightarrow n+\nu$. The inverse process is ineffective. This process ensures that $x_{1} \ll 1$, and $P_{1 s}, P_{2 p 1 s} \simeq 1$

The atomic states are short-lived and we may estimate $x_{1}$ and $x_{2}$ by putting $\dot{x_{1}}=$ $\dot{x_{2}}=0$, which we call $x_{1,0}$ and $x_{2,0}$. We find that $x_{2,0} \ll x_{1,0}$ during recombination where $T \ll E_{2 s 1 s}$, and the evolution equation of $x_{e}$ is given by

$$
\dot{x_{e}}=-x_{1,0} \Gamma_{1 s, e c}-x_{2,0} \Gamma_{2 s, e c} \simeq-x_{1,0} \Gamma_{1 s, e c} .
$$

The full expression for $x_{1,0}$ is not simple, but we can find an approximate solution by adding eqs. (E.3) and (E.4), and neglecting $x_{2}$,

$$
x_{1,0} \simeq \frac{x_{e}^{2} n_{\mathrm{DM}}\left(\alpha_{1}+\alpha_{B}\right)}{\beta_{1}+\Gamma_{1 s, e c}} .
$$


The evolution equation of $x_{e}$ is given by

$$
\dot{x_{e}} \simeq-x_{e}^{2} n_{\mathrm{DM}}\left(\alpha_{1}+\alpha_{B}\right) \frac{\Gamma_{1 s, e c}}{\beta_{1}+\Gamma_{1 s, e c}} .
$$

This equation has a simple interpretation. Once the mirror electron is recombined into atomic states, it rapidly falls into the ground state. The total rate of the formation of the ground state is given by $x_{e}^{2} n_{\mathrm{DM}}\left(\alpha_{1}+\alpha_{B}\right)$. The ground state mirror electron is again scattered into a free state with a rate $\beta_{1}$ or is captured by the mirror proton with a rate $\Gamma_{1 s, e c}$. The latter contributes to recombination, and hence the recombination rate is suppressed by $\Gamma_{1 s, e c} /\left(\beta_{1}+\Gamma_{1 s, e c}\right)$.

A sample evolution of the ionization fraction of the mirror electron is shown in figure 15 . Here we use the full expression for $x_{1,0}$. An approximated $x_{1,0}$ gives about a $10 \%$ larger ionization fraction. In the calculation we take $v^{\prime} / v=4$ and $m_{p}+m_{n}-m_{e}=m_{e} / 2$ to estimate the mirror electron capture rate, but the resultant ionization fraction is insensitive to these parameters, since during recombination $\beta_{1} \ll \Gamma_{1 s, e c}$ and the dependence on $\Gamma_{1 s, e c}$ drops out from eq. (E.12).

Open Access. This article is distributed under the terms of the Creative Commons Attribution License (CC-BY 4.0), which permits any use, distribution and reproduction in any medium, provided the original author(s) and source are credited.

\section{References}

[1] T.D. Lee and C.-N. Yang, Question of Parity Conservation in Weak Interactions, Phys. Rev. 104 (1956) 254 [INSPIRE].

[2] I. Yu. Kobzarev, L.B. Okun and I. Ya. Pomeranchuk, On the possibility of experimental observation of mirror particles, Sov. J. Nucl. Phys. 3 (1966) 837 [Yad. Fiz. 3 (1966) 1154] [INSPIRE].

[3] H. Goldberg and L.J. Hall, A New Candidate for Dark Matter, Phys. Lett. B 174 (1986) 151 [INSPIRE].

[4] Z. Chacko, H.-S. Goh and R. Harnik, The Twin Higgs: Natural electroweak breaking from mirror symmetry, Phys. Rev. Lett. 96 (2006) 231802 [hep-ph/0506256] [INSPIRE].

[5] R. Barbieri, L.J. Hall and K. Harigaya, Minimal Mirror Twin Higgs, JHEP 11 (2016) 172 [arXiv: 1609.05589] [INSPIRE].

[6] N. Craig, S. Koren and T. Trott, Cosmological Signals of a Mirror Twin Higgs, JHEP 05 (2017) 038 [arXiv: 1611.07977] [inSPIRE].

[7] Z. Chacko, N. Craig, P.J. Fox and R. Harnik, Cosmology in Mirror Twin Higgs and Neutrino Masses, JHEP 07 (2017) 023 [arXiv: 1611.07975] [INSPIRE].

[8] R. Barbieri, T. Gregoire and L.J. Hall, Mirror world at the large hadron collider, hep-ph/0509242 [INSPIRE].

[9] C.D. Froggatt and H.B. Nielsen, Hierarchy of Quark Masses, Cabibbo Angles and CP-violation, Nucl. Phys. B 147 (1979) 277 [INSPIRE].

[10] D.E. Kaplan and T.M.P. Tait, New tools for fermion masses from extra dimensions, JHEP 11 (2001) 051 [hep-ph/0110126] [INSPIRE]. 
[11] P. Batra and Z. Chacko, A Composite Twin Higgs Model, Phys. Rev. D 79 (2009) 095012 [arXiv:0811.0394] [INSPIRE].

[12] M. Geller and O. Telem, Holographic Twin Higgs Model, Phys. Rev. Lett. 114 (2015) 191801 [arXiv: 1411.2974] [INSPIRE].

[13] R. Barbieri, D. Greco, R. Rattazzi and A. Wulzer, The Composite Twin Higgs scenario, JHEP 08 (2015) 161 [arXiv: 1501.07803] [INSPIRE].

[14] M. Low, A. Tesi and L.-T. Wang, Twin Higgs mechanism and a composite Higgs boson, Phys. Rev. D 91 (2015) 095012 [arXiv:1501.07890] [INSPIRE].

[15] H.-C. Cheng, S. Jung, E. Salvioni and Y. Tsai, Exotic Quarks in Twin Higgs Models, JHEP 03 (2016) 074 [arXiv: 1512.02647] [INSPIRE].

[16] C. Csáki, M. Geller, O. Telem and A. Weiler, The Flavor of the Composite Twin Higgs, JHEP 09 (2016) 146 [arXiv: 1512.03427] [INSPIRE].

[17] H.-C. Cheng, E. Salvioni and Y. Tsai, Exotic electroweak signals in the twin Higgs model, Phys. Rev. D 95 (2017) 115035 [arXiv:1612.03176] [INSPIRE].

[18] R. Contino, D. Greco, R. Mahbubani, R. Rattazzi and R. Torre, Precision Tests and Fine Tuning in Twin Higgs Models, arXiv: 1702.00797 [INSPIRE].

[19] A. Falkowski, S. Pokorski and M. Schmaltz, Twin SUSY, Phys. Rev. D 74 (2006) 035003 [hep-ph/0604066] [INSPIRE].

[20] S. Chang, L.J. Hall and N. Weiner, A supersymmetric twin Higgs, Phys. Rev. D 75 (2007) 035009 [hep-ph/0604076] [INSPIRE].

[21] N. Craig and K. Howe, Doubling down on naturalness with a supersymmetric twin Higgs, JHEP 03 (2014) 140 [arXiv: 1312.1341] [INSPIRE].

[22] A. Katz, A. Mariotti, S. Pokorski, D. Redigolo and R. Ziegler, SUSY Meets Her Twin, JHEP 01 (2017) 142 [arXiv:1611.08615] [INSPIRE].

[23] M. Badziak and K. Harigaya, Supersymmetric D-term Twin Higgs, JHEP 06 (2017) 065 [arXiv: 1703.02122] [INSPIRE].

[24] N. Arkani-Hamed and M. Schmaltz, Hierarchies without symmetries from extra dimensions, Phys. Rev. D 61 (2000) 033005 [hep-ph/9903417] [InSPIRE].

[25] K.S. Babu, T. Enkhbat and I. Gogoladze, Anomalous U(1) symmetry and lepton flavor violation, Nucl. Phys. B 678 (2004) 233 [hep-ph/0308093] [INSPIRE].

[26] K.S. Babu and T. Enkhbat, Fermion mass hierarchy and electric dipole moments, Nucl. Phys. B 708 (2005) 511 [hep-ph/0406003] [inSPIRE].

[27] K.S. Babu, A. Khanov and S. Saad, Anarchy with Hierarchy: A Probabilistic Appraisal, Phys. Rev. D 95 (2017) 055014 [arXiv: 1612.07787] [INSPIRE].

[28] N. Craig, A. Katz, M. Strassler and R. Sundrum, Naturalness in the Dark at the LHC, JHEP 07 (2015) 105 [arXiv: 1501.05310] [INSPIRE].

[29] S. Antusch and V. Maurer, Running quark and lepton parameters at various scales, JHEP 11 (2013) 115 [arXiv: 1306.6879] [INSPIRE].

[30] CP-PACS collaboration, M. Okamoto et al., Equation of state for pure SU(3) gauge theory with renormalization group improved action, Phys. Rev. D 60 (1999) 094510 [hep-lat/9905005] [INSPIRE]. 
[31] ATLAS and CMS collaborations, Measurements of the Higgs boson production and decay rates and constraints on its couplings from a combined ATLAS and CMS analysis of the LHC pp collision data at $\sqrt{s}=7$ and $8 \mathrm{TeV}, J H E P 08$ (2016) 045 [arXiv:1606.02266] [INSPIRE].

[32] Z. Berezhiani, D. Comelli and F.L. Villante, The early mirror universe: Inflation, baryogenesis, nucleosynthesis and dark matter, Phys. Lett. B 503 (2001) 362 [hep-ph/0008105] [INSPIRE].

[33] A. Yu. Ignatiev and R.R. Volkas, Mirror dark matter and large scale structure, Phys. Rev. D 68 (2003) 023518 [hep-ph/0304260] [INSPIRE].

[34] H. Fukuda, K. Harigaya, M. Ibe and T.T. Yanagida, Model of visible QCD axion, Phys. Rev. D 92 (2015) 015021 [arXiv: 1504.06084] [INSPIRE].

[35] H. Fukuda, M. Ibe and T.T. Yanagida, Dark Matter Candidates in a Visible Heavy QCD Axion Model, Phys. Rev. D 95 (2017) 095017 [arXiv: 1702.00227] [INSPIRE].

[36] H. Davoudiasl and R.N. Mohapatra, On Relating the Genesis of Cosmic Baryons and Dark Matter, New J. Phys. 14 (2012) 095011 [arXiv:1203.1247] [InSPIRE].

[37] K. Petraki and R.R. Volkas, Review of asymmetric dark matter, Int. J. Mod. Phys. A 28 (2013) 1330028 [arXiv:1305.4939] [INSPIRE].

[38] K.M. Zurek, Asymmetric Dark Matter: Theories, Signatures and Constraints, Phys. Rept. 537 (2014) 91 [arXiv: 1308.0338] [INSPIRE].

[39] M. Farina, A. Monteux and C.S. Shin, Twin mechanism for baryon and dark matter asymmetries, Phys. Rev. D 94 (2016) 035017 [arXiv: 1604.08211] [INSPIRE].

[40] N. Craig and A. Katz, The Fraternal WIMP Miracle, JCAP 10 (2015) 054 [arXiv: 1505.07113] [INSPIRE].

[41] I. Garcia Garcia, R. Lasenby and J. March-Russell, Twin Higgs Asymmetric Dark Matter, Phys. Rev. Lett. 115 (2015) 121801 [arXiv: 1505. 07410] [INSPIRE].

[42] M. Farina, Asymmetric Twin Dark Matter, JCAP 11 (2015) 017 [arXiv:1506.03520] [INSPIRE].

[43] XENON collaboration, E. Aprile et al., First Dark Matter Search Results from the XENON1T Experiment, arXiv:1705.06655 [INSPIRE].

[44] XENON collaboration, E. Aprile et al., Physics reach of the XENON1T dark matter experiment, JCAP 04 (2016) 027 [arXiv: 1512.07501] [INSPIRE].

[45] LZ collaboration, D.S. Akerib et al., LUX-ZEPLIN (LZ) Conceptual Design Report, arXiv: 1509.02910 [INSPIRE].

[46] DARWIN collaboration, J. Aalbers et al., DARWIN: towards the ultimate dark matter detector, JCAP 11 (2016) 017 [arXiv:1606.07001] [INSPIRE].

[47] J. Billard, L. Strigari and E. Figueroa-Feliciano, Implication of neutrino backgrounds on the reach of next generation dark matter direct detection experiments, Phys. Rev. D 89 (2014) 023524 [arXiv: 1307.5458] [inSPIRE].

[48] S. Seager, D.D. Sasselov and D. Scott, A new calculation of the recombination epoch, Astrophys. J. 523 (1999) L1 [astro-ph/9909275] [INSPIRE].

[49] M. Markevitch et al., Direct constraints on the dark matter self-interaction cross-section from the merging galaxy cluster 1E065\%-56, Astrophys. J. 606 (2004) 819 [astro-ph/0309303] [INSPIRE]. 
[50] S.W. Randall, M. Markevitch, D. Clowe, A.H. Gonzalez and M. Bradac, Constraints on the Self-Interaction Cross-Section of Dark Matter from Numerical Simulations of the Merging Galaxy Cluster 1E 0657-56, Astrophys. J. 679 (2008) 1173 [arXiv:0704.0261] [INSPIRE].

[51] R.A. Aziz and V.P.S. Nain, An accurate intermolecular potential for helium, J. Chem. Phys. 70 (1979) 4330.

[52] M. Kaplinghat, S. Tulin and H.-B. Yu, Dark Matter Halos as Particle Colliders: Unified Solution to Small-Scale Structure Puzzles from Dwarfs to Clusters, Phys. Rev. Lett. 116 (2016) 041302 [arXiv: 1508.03339] [INSPIRE].

[53] J.M. Cline, Z. Liu, G. Moore and W. Xue, Scattering properties of dark atoms and molecules, Phys. Rev. D 89 (2014) 043514 [arXiv: 1311.6468] [INSPIRE].

[54] I. Affleck and M. Dine, A New Mechanism for Baryogenesis, Nucl. Phys. B 249 (1985) 361 [INSPIRE].

[55] M. Dine, L. Randall and S.D. Thomas, Baryogenesis from flat directions of the supersymmetric standard model, Nucl. Phys. B 458 (1996) 291 [hep-ph/9507453] [INSPIRE].

[56] G. Krnjaic and K. Sigurdson, Big Bang Darkleosynthesis, Phys. Lett. B 751 (2015) 464 [arXiv: 1406.1171] [INSPIRE].

[57] E. Hardy, R. Lasenby, J. March-Russell and S.M. West, Big Bang Synthesis of Nuclear Dark Matter, JHEP 06 (2015) 011 [arXiv:1411.3739] [INSPIRE].

[58] NPLQCD collaboration, S.R. Beane et al., Light Nuclei and Hypernuclei from Quantum Chromodynamics in the Limit of SU(3) Flavor Symmetry, Phys. Rev. D 87 (2013) 034506 [arXiv: 1206.5219] [INSPIRE].

[59] T. Yamazaki, K.-i. Ishikawa, Y. Kuramashi and A. Ukawa, Helium nuclei, deuteron and dineutron in 2+1 flavor lattice QCD, Phys. Rev. D 86 (2012) 074514 [arXiv:1207.4277] [INSPIRE].

[60] S. Borsányi et al., Calculation of the axion mass based on high-temperature lattice quantum chromodynamics, Nature $\mathbf{5 3 9}$ (2016) 69 [arXiv:1606.07494] [INSPIRE].

[61] A. Sommerfeld, Über die Beugung und Bremsung der Elektronen, Annalen Phys. 403 (1931) 257.

[62] S. Cassel, Sommerfeld factor for arbitrary partial wave processes, J. Phys. G 37 (2010) 105009 [arXiv: 0903.5307] [INSPIRE].

[63] T. Nagano, A. Ota and Y. Sumino, $O\left(\alpha_{s}^{2}\right)$ corrections to $e^{+} e^{-} \rightarrow t \bar{t}$ total and differential cross-sections near threshold, Phys. Rev. D 60 (1999) 114014 [hep-ph/9903498] [INSPIRE].

[64] H.B. Meyer, Glueball matrix elements: A lattice calculation and applications, JHEP 01 (2009) 071 [arXiv:0808.3151] [INSPIRE].

[65] Y. Chen et al., Glueball spectrum and matrix elements on anisotropic lattices, Phys. Rev. D 73 (2006) 014516 [hep-lat/0510074] [INSPIRE].

[66] F. Feruglio, Pieces of the Flavour Puzzle, Eur. Phys. J. C 75 (2015) 373 [arXiv: 1503.04071] [INSPIRE].

[67] P.J.E. Peebles, Recombination of the Primeval Plasma, Astrophys. J. 153 (1968) 1 [INSPIRE].

[68] D. Pequignot, P. Petitjean and C. Boisson, Total and effective radiative recombination coefficients, Astron. Astrophys. 251 (1991) 680. 